\title{
OSTEOGENIC DIFFERENTIATION OF HUMAN ADIPOSE-DERIVED STEM CELLS IN 3D CONDITIONS - COMPARISON OF SPHEROIDS AND POLYSTYRENE SCAFFOLDS
}

\author{
S. Rumiński ${ }^{1,2,3}$, I. Kalaszczyńska ${ }^{1,2}$, A. Długosz ${ }^{2,4}$ and M. Lewandowska-Szumieł ${ }^{1,2,4, *}$ \\ ${ }^{1}$ Department of Histology and Embryology, Centre for Biostructure Research, \\ Medical University of Warsaw, Warsaw, Poland \\ ${ }^{2}$ Centre for Preclinical Research and Technology, Warsaw, Poland \\ ${ }^{3}$ Postgraduate School of Molecular Medicine, Medical University of Warsaw, Warsaw, Poland \\ ${ }^{4}$ Laboratory for Cell Research and Application, Medical University of Warsaw, Warsaw, Poland
}

\begin{abstract}
Expansion and differentiation of adipose-derived stem cells (ADSCs) in vitro are routinely performed in two-dimensional (2D) environments. The study hypothesis was that the utilisation of three-dimensional (3D) culture conditions, mimicking the natural stem cell niche, might increase the osteogenic commitment of ADSCs. Therefore, human ADSCs were seeded in 3D culture systems lacking bioactive material components: spheroids and polystyrene scaffolds. Alkaline phosphatase (ALP) activity, a marker of early osteogenesis, was higher in ADSC spheroids and ADSC seeded on polystyrene scaffolds as compared to 2D cultures. Furthermore, the expression of the osteoblast marker genes Runt-related transcription factor 2 (RUNX2), osterix and integrin binding sialoprotein $(I B S P)$ was significantly up-regulated in spheroids as compared to polystyrene scaffolds and 2D culture. Elevated levels of RUNX2 and IBSP in spheroids were confirmed at the protein level by Western blot and immunofluorescence, respectively. Bone mineral production was lower in spheroids than in polystyrene scaffolds and 2D culture at day 14. Curiously, adipocyte differentiation was downregulated in spheroids as compared to 2D-culture. Finally, to induce late differentiation events, cells were dissociated from spheroids after a $7 \mathrm{~d}$ osteogenic pre-differentiation culture and replated in 2D culture in osteoblast maturation medium. After a subsequent $14 \mathrm{~d}$ of maturation, cells produced bone mineral and osteocalcin proteins, which are late osteoblast markers.

The present work showed that the 3D environment may provide additional stimuli for the commitment of ADSCs to the osteogenic lineage. Furthermore, the presented data may be valuable when designing protocols to prepare ADSCs for use in bone regeneration clinical studies.
\end{abstract}

Keywords: 3D culture, osteoblast, osteogenic differentiation, mesenchymal stem cells, bone regeneration, adipose derived stem cells, adipose tissue.

*Address for correspondence: Prof. M. Lewandowska-Szumieł, Medical University of Warsaw, Warsaw, Poland.

Email: mszumiel@wum.edu.pl

Copyright policy: This article is distributed in accordance with Creative Commons Attribution Licence (http://creativecommons.org/licenses/by-sa/4.0/).

\section{Introduction}

Mesenchymal stem cells (MSCs) have become the first-choice cell source for research and therapeutic applications in the field of regenerative medicine. Since their first recognition by Pittenger et al. (1999), multiple tissues have been characterised as a source of MSCs. The first studies, including the work by Pittenger, were performed on bone-marrow-derived MSCs (BMSCs). However, shortly after, MSCs were successfully isolated from several human tissues, including adipose tissue (Zuk et al., 2001), umbilical cord blood (Erices et al., 2000; Goodwin et al., 2001) and Wharton's jelly of the umbilical cord (Romanov et al., 2003; Wang et al., 2004). Currently, BMSCs and adipose-derived mesenchymal stem cells (ADSCs) are recognised as the most well-studied MSC types (Tabatabaei Qomi and Sheykhhasan, 2017). Both BMSCs and ADSCs are capable of multilineage differentiation, including differentiation into osteoblasts. The osteogenic differentiation capability of MSCs is widely utilised in bone tissue engineering, 
where MSCs serve as bone-regenerating and bonereplacing agents. Compared to BMSCs, ADSCs have several advantages, such as low-morbidity associated with tissue collection, high yield, high proliferation rate and efficient growth factor production, that make them favoured for clinical application (Paduano et al., 2017; Tabatabaei Qomi and Sheykhhasan, 2017). However, ADSCs differentiate into osteoblasts in vitro less efficiently than BMSCs (Shafiee et al., 2011; Vishnubalaji et al., 2012).

The mechanisms regulating ADSC osteogenic commitment are far less understood than the mechanisms behind BMSCs (Paduano et al., 2017). Nonetheless, several pathways regulating ADSC osteogenesis have been identified, such as the extracellular signal-related kinase (ERK) (Liu et al., 2009), Wnt/ $\beta$-catenin (Guidotti et al., 2013; Hsu and Huang, 2013), tumour necrosis factor (TNF)/ nuclear factor (NF)-kB (Cho et al., 2010) and Notch pathways (Lough et al., 2016). The central point of this intricate regulatory network is the Runt-related transcription factor 2 (RUNX2), which drives the osteogenic differentiation of MSCs. Following RUNX2 activation, several downstream genes are activated, including the transcription factor osterix, alkaline phosphatase (ALP) and genes encoding bone matrix proteins, such as collagen type I, osteonectin, bone sialoprotein (BSP) and osteocalcin (Liu and Lee, 2013)

Three-dimensional (3D) scaffolds designed for bone engineering applications can be manufactured from a variety of biomaterials including, but not limited to, calcium phosphates, polycaprolactone, hyaluronic acid, hydroxyapatite, collagen and fibroin. A common feature of well-designed scaffolds is their microporous architecture, which allows efficient seeding with stem cells in vitro and ingrowth of host tissue in vivo (Shakya and Kandalam, 2017). The 3D scaffold itself provides a microenvironment that, ideally, should support the formation of bone tissue by the in-vitro-introduced cells. The components and factors of the 3D microenvironment (extracellular matrix proteins, growth factors and released ions) or the material surface properties (hydrophobicity, charge, topography, etc.) may exert biological effects on the seeded stem cells (Hao et al., 2017). These effects include induction of osteogenic differentiation. Therefore, it is not surprising that several authors have reported a beneficial effect of the applied 3D scaffold on MSC osteogenesis. Hishikawa et al. (2004) showed that for human BMSCs, culture in 3D scaffolds made from collagen or synthetic copolymers [poly(N-isopropylacrylamide) and poly(ethylene glycol)] stimulates more mineralisation, ALP activity and osteocalcin production than monolayer culture. Similarly, Gabbay et al. (2006) reported up-regulated expression of several osteogenic marker genes in ADSCs cultured in a 3D collagen gel as compared to $2 \mathrm{D}$ culture. More recent data have shown that a hydroxyapatite/ $\beta$-tricalcium phosphate scaffold can induce osteogenic marker gene expression in BMSCs cultured in medium without osteogenic supplements (Mebarki et al., 2017), which proves the osteoinductive properties of the tested scaffold.

Several authors reported improved osteogenic differentiation in systems where the osteoinductive effect might be solely dependent on the 3D architecture of the scaffold. Li et al. (2017) reported elevated ALP activity and mineral deposition by ADSCs cultured on reduced graphene oxide/polydimethylsiloxane 3D scaffolds (3D RGO/PDMS) as compared to 2D RGO/PDMS. Furthermore, both BMSCs and ADSCs demonstrate up-regulated expression of the osteogenic markers ALP, BMP-2, RUNX2 and COL1A1 on polycaprolactone (PCL) nanofibre scaffolds as compared to standard 2D culture (Xue et al., 2017). Finally, studies on 3D-printed PCL/ tricalcium phosphate (TCP) scaffolds provided evidence for enhanced osteogenesis of seeded ADSCs as compared to 2D culture (Rumiński et al., 2018). Surprisingly, the presence of TCP is not responsible for the osteoinductive effect. As polycaprolactone itself is neutral for MSC osteogenesis (Romagnoli et al., 2015), the improvement in differentiation efficiency (Xue et al., 2017 should presumably be attributed to the $3 \mathrm{D}$ environment provided by the scaffolds.

To elucidate if the three-dimensionality of a culture system may significantly influence the differentiation of ADSCs, commercially available polystyrene scaffolds (Knight and Przyborski, 2015; MacDonald et al., 2014) were applied as a model providing 3D architecture and allowing cell adhesion without exerting a specific biological effect.

In addition to many scaffold-based systems, spheroid culture offers another approach for culturing MSCs in 3D that compensates for many of the deficiencies seen in monolayer cultures. Spheroids are multicellular aggregates that grow in vitro in unattached spherical structures. MSCs can easily be induced to form spheroids by applying the hanging drop technique or by seeding cells on to non-adherent surfaces (Fennema et al., 2013). Several authors have already reported improved osteogenesis of cells in vitro in spheroids. Kale et al. (2000) showed an elevated level of osteonectin, collagen type I and ALP in spheroids of MG-63 cells, human bone precursor cells and human primary osteoblasts. Similarly, up-regulated expression of marker genes - osterix, RUNX2, osteopontin $(O P N)$ and BSP - in rat BMSCs is observed in spheroid cultures (Yamaguchi et al., 2014). Others have reported up-regulated osteogenic events in short-term attachment of ADSC spheroids generated on chitosan-containing surfaces (Cheng et al., 2012; Hsu and Huang, 2013) or in BMSC spheroids attached to a micropatterned collagen/ photosensitised poly(ethylene glycol) (P-PEG) surface (Wang et al., 2009).

In the present study, spheroids generated on nonadhesive surfaces were studied without providing any external attachment substrate throughout the investigated culture period, to elucidate the role 
of a cell-derived spheroid microenvironment on ADSC osteogenic commitment. Therefore, the study described the impact of the two selected 3D culture models on the efficiency of ADSC early and late osteogenic differentiation events.

\section{Materials and Methods}

\section{Cell isolation and expansion}

Human adipose tissue samples were obtained during cosmetic liposuction and in agreement with the local bioethics committee (approval KB/85/A/2012). Adipose tissue collected from eight donors was processed to provide cells for all experiments. The isolation procedure was conducted using a wellcharacterised method (Zuk et al., 2001). Briefly, adipose tissue was washed three times with phosphate-buffered saline (PBS) and incubated with collagenase type XI (400 U/mL, Sigma-Aldrich) at $37^{\circ} \mathrm{C}$ for $60 \mathrm{~min}$, while agitating. Afterward, the digested lipoaspirate was centrifuged at $300 \times g$ for $10 \mathrm{~min}$ and the upper lipid phase was discarded. The pelleted stromal vascular fraction (SVF) was resuspended in complete medium (CM) composed of Dulbecco's modified eagle medium (DMEM), $10 \%$ foetal bovine serum (FBS), $1 \%$ antibiotic-antimycotic (Life Technologies) and $5 \mathrm{ng} / \mathrm{mL}$ recombinant human fibroblast growth factor-2 (FGF-2; Sigma-Aldrich) and filtered using a $100 \mu \mathrm{m}$ filter mesh (Becton Dickinson). Then, cells were seeded into T-75 culture flasks at a density of $3 \times 10^{6}$ nucleated cells per flask and cultured at $37^{\circ} \mathrm{C}$ and $5 \% \mathrm{CO}_{2}$ in a humidified atmosphere. After reaching confluence, which occurred 4-7 d after seeding, the obtained ADSCs were detached using trypsin and cryopreserved. Cells at passage 2 or 3 were used for experiments. Each experiment was conducted on cells obtained from three or four donors, as specified below. A cell population from a single donor was processed and studied independently from the other donor-specific cell populations. Each experiment was performed on cell cultures derived from a common set of donors.

\section{D culture and osteogenic differentiation}

Osteogenic differentiation medium (OM) was composed of CM without FGF-2 and supplemented with $10 \mathrm{nM}$ dexamethasone, $50 \mu \mathrm{g} / \mathrm{mL}$ ascorbic acid 2-phosphate and $3 \mathrm{mM} \mathrm{NaH} \mathrm{PO}_{4}$ (all from SigmaAldrich). In osteoblast maturation experiments, the $\mathrm{OM}$ was further supplemented with $10 \mathrm{nM} 1 \alpha, 25-$ dihydroxyvitamin $\mathrm{D}_{3}$ (OM-D3).

Spheroids were generated using a method adapted from Hildebrandt et al. (2011). A $10 \% \mathrm{w} / \mathrm{v}$ solution of Pluronic ${ }^{\circledR}$ F-127 (Sigma-Aldrich) in deionised water was prepared and sterile-filtered through a $0.22 \mu \mathrm{m}$ syringe filter. Next, the solution was added to the wells of a 96-well sterile round-bottom culture plate (ThermoFisher Scientific). Excess solution was aspirated, and the plate was left to dry overnight at $37^{\circ} \mathrm{C}$. Afterward, the plate was washed once with PBS and $200 \mu \mathrm{L}$ of cell suspension, prepared in either CM or OM, was added to each well. The concentration of cells in the suspension was adjusted to seed 10,000 viable cells per well. The plate was kept in the cell culture incubator on a rotary shaker under gentle rotation. The medium was renewed every 2-3 d, with approximately $75 \%$ of the culture volume replaced with fresh medium.

As a 3D scaffold model, commercially available 3D porous polystyrene (PS) scaffolds [Alvetex ${ }^{\circledR}$ 12-well inserts (ReproCELL Europe, Glasgow, UK)] were utilised. The scaffolds were inserted into standard 6-well culture plates (ThermoFisher Scientific) and submerged in $70 \%$ ethanol for $1 \mathrm{~h}$. Next, the scaffold was rinsed thoroughly with sterile PBS. Then, $100 \mu \mathrm{L}$ of a suspension containing $1 \times 10^{6}$ cells $/ \mathrm{mL}$ in $\mathrm{OM}$ were applied and the scaffold was placed in the cell culture incubator for $40 \mathrm{~min}$ to allow cell attachment. Finally, the scaffold was submerged in OM. The medium was replaced every 2-3 d.

To prepare 2D monolayer cultures (2D tissue culture polystyrene, 2D TCPS), cells were seeded into standard 6-well or 24-well culture plates (ThermoFisher Scientific) in either CM, OM or OMD3.

\section{Cell viability and number}

Cells were cultured for 2 to $14 \mathrm{~d}$ in $\mathrm{OM}$ in all three culture systems. Staining with fluorescein diacetate (Life Technologies) and propidium iodide (SigmaAldrich) was performed to visualise viable and dead cells. Then, cells were observed with a Nikon Eclipse-Ti inverted microscope (Nikon Instruments Inc.). Images were acquired at different focal planes (z-stack) and processed with Nikon NIS software.

For quantification of the relative cell number, the spheroids were collected in culture medium and centrifuged. Spheroid pellet, 2D TCPS and polystyrene scaffold cultures were washed with PBS and lysed with $0.1 \%$ Triton-X (Sigma-Aldrich) in PBS. Next, suspensions were frozen at $-80{ }^{\circ} \mathrm{C}$. Before analysis, the lysates were subjected to five freeze-thaw cycles. DNA concentration in the lysates was determined by Quant-iT ${ }^{\mathrm{TM}}$ PicoGreen ${ }^{\mathrm{TM}}$ dsDNA Assay, according to the manufacturer's instructions (ThermoFisher Scientific). A reference sample was prepared from a known number of cells $\left(5 \times 10^{5}\right)$ from each patient and collected at the time of culture initiation. Fluorescence of the PicoGreen dye was measured with a FLUOstar plate reader (BMG Labtech GMBH, Ortenberg, Germany). Then, DNA content in the tested cultures was normalised to DNA content in the reference sample. Experiments were performed on cells isolated from three donors.

\section{Cell proliferation analysis}

ADSCs were seeded in CM containing FGF-2 or in OM into 2D TCPS, 3D spheroids or 3D PS scaffolds, as 
described above. Cells were cultured for 2, 7 and $14 \mathrm{~d}$; however, the latter two time points included only the OM-cultured ADSCs. At the time of analysis, cells in all cultures were pulsed with 5-ethynyl-2'-deoxyuridine (EdU) for $2 \mathrm{~h}$ to label cells in the $\mathrm{S}$ phase of the cell cycle. Next, cultures were treated with Accumax (Sigma-Aldrich) to obtain a single cell suspension. Then, cell samples were fixed and the DNAincorporated EdU was labelled with AlexaFluor ${ }^{\mathrm{TM}}$ 488 azide, using the Click-iT ${ }^{\circledR}$ EdU Flow Cytometry Cell Proliferation Assay (ThermoFisher Scientific), according to the manufacturer's instructions. Stained cells were evaluated on a CytoFLEX flow cytometer (Beckman Coulter Inc., Indianapolis, IN, USA). Data acquired from single-stained cell samples and from an unlabelled cell sample were used for fluorescence compensation calculations. Acquired data were analysed with FlowJo software (FlowJo, LLC, Ashland, OR, USA). EdU-positive cells were gated to determine the percentage of cells undergoing DNA replication in relation to all cells in the sample. Experiments were performed on cells isolated from four donors. Samples from a single donor were acquired in duplicate.

\section{ALP activity}

ALP activity was measured in $0.1 \%$ Triton- $X$ cell lysates obtained as described above. An ALP substrate, p-nitrophenyl phosphate (Sigma-Aldrich), was added to the cell lysates and the solution was incubated for $30 \mathrm{~min}$ at room temperature. Then, the reaction was stopped with $3 \mathrm{M} \mathrm{NaOH}$ and the absorbance of the yellow product was read at $485 \mathrm{~nm}$ with the FLUOstar plate reader (BMG Labtech). The concentration of the product was calculated from a standard curve generated using serial dilutions of 4-nitrophenol (Sigma-Aldrich). The enzymatic activity was expressed as $\mu \mathrm{mol}$ of 4 -nitrophenol produced per min, normalised to the content of DNA in the sample. Values were presented relative to the enzymatic activity of the undifferentiated control ADSC sample. Experiments were performed on cells isolated from three donors.

\section{Gene expression analysis}

Cells cultured in OM in spheroids, polystyrene scaffolds or 2D TCPS were lysed with Fenzol buffer (A\&A Biotechnology, Gdynia, Poland). The lysates were stored at $-80{ }^{\circ} \mathrm{C}$ until analysis. Total RNA was isolated with the Total RNA Mini Plus Concentrator Kit (A\&A Biotechnology), according to the manufacturer's instructions. RNA concentration and purity were determined with a NanoDrop Spectrophotometer (ThermoFisher Scientific). RNase-free DNase was used to remove possible contamination of genomic DNA (A\&A Biotechnology). To obtain complementary deoxyribose nucleic acid (cDNA), 600 ng of RNA was reverse-transcribed with a High Capacity cDNA Synthesis Kit (ThermoFisher Scientific) on a 7500 Fast thermal cycler (Applied Biosystems, Carlsbad,
CA, USA). The real-time polymerase chain reaction (PCR) was carried out with the prepared cDNA as a template and TaqMan ${ }^{\circledR}$ Universal PCR Master Mix (ThermoFisher Scientific). Expression level of RUNX2 Hs00231692_m1), osterix (SP7, Hs01866874_s1), collagen type I alpha 1 (COL1A1, Hs01029144_m1), integrin binding sialoprotein (IBSP, Hs00173720_m1), bone gamma-carboxyglutamic acid-containing protein (BGLAP; osteocalcin, Hs01587814_g1), peroxisome proliferator-activated receptor gamma (PPARG, Hs01115513_m1), fatty acid binding protein 4 (FABP4, Hs01086177_m1), CCAAT/enhancer binding protein alpha (CEBPA, Hs00269972_s1) and lipoprotein lipase (LPL, Hs00173425_m1) genes was analysed using TaqMan ${ }^{\circledR}$ Gene Expression Assays (ThermoFisher Scientific). Detection of each transcript was performed in triplicate. Relative gene expression was determined using the comparative $\Delta \Delta \mathrm{Ct}$ method using ubiquitin C gene (UBC, Hs00824723_m1) as a reference. Undifferentiated ADSCs derived from the same cell population as used for treatment conditions but cultured for $2 \mathrm{~d}$ in $\mathrm{CM}$ were used as reference samples. Experiments were performed on cells isolated from three donors.

\section{Western blot}

For Western blot analysis, cells cultured on 2D TCPS, spheroids and scaffolds were lysed with radioimmunoprecipitation assay (RIPA) buffer (ThermoFisher Scientific). Total protein concentration was analysed with Pierce BCA assay (ThermoFisher Scientific) following manufacturer's instructions. Absorbance measurement after the reaction was performed with FLUOstar plate reader (BMG Labtech). Cell lysates were mixed with Laemmli sample buffer containing $10 \% \mathrm{v} / \mathrm{v}$ beta-mercaptoethanol. Prepared samples were loaded on $10 \%$ sodium dodecyl sulphate-polyacrylamide gel (National Diagnostics, Atlanta, GA, USA), with the volume of the sample loaded corresponding to $10 \mu \mathrm{g}$ of total protein. Electrophoresis was run using the MiniProtean Electrophoresis Set (BioRad Laboratories). Proteins were blotted on to an immobilon-P membranes (Merck Millipore) using a semi-dry blotting apparatus (ThermoFisher Scientific). The membrane was blocked with $5 \%$ skimmed milk in Tris-buffered saline and incubated with rabbit polyclonal anti-Runx2 antibody (ThermoFisher Scientific) or mouse monoclonal anti- $\beta$-actin antibody (Merck Millipore). After washing, the membrane was incubated with anti-rabbit or anti-mouse antibody conjugated with horseradish peroxidase (both ThermoFisher Scientific). Finally, the membrane was incubated in enhanced chemiluminescent (ECL) horseradish peroxidase substrate (ThermoFisher Scientific) and protein bands were detected on a photographic film (Sigma-Aldrich). Densitometric analysis of blot images was performed with ImageJ software (Schneider et al., 2012). Experiments were performed on cells isolated from three donors. 


\section{Bone mineral staining}

Cells were seeded and cultured in OM in spheroids and polystyrene scaffolds as described above or on TCPS 24-well plates for the monolayer culture. Cells were fixed in $10 \%$ buffered formalin (Sigma-Aldrich) and stained with OsteoImage $\mathrm{T}^{\mathrm{TM}}$ (Lonza), according to the manufacturer's instructions, to detect bone minerals. Cell nuclei were counterstained with Hoechst 33342 (Sigma-Aldrich). Undifferentiated ADSCs were used as a negative control for staining.
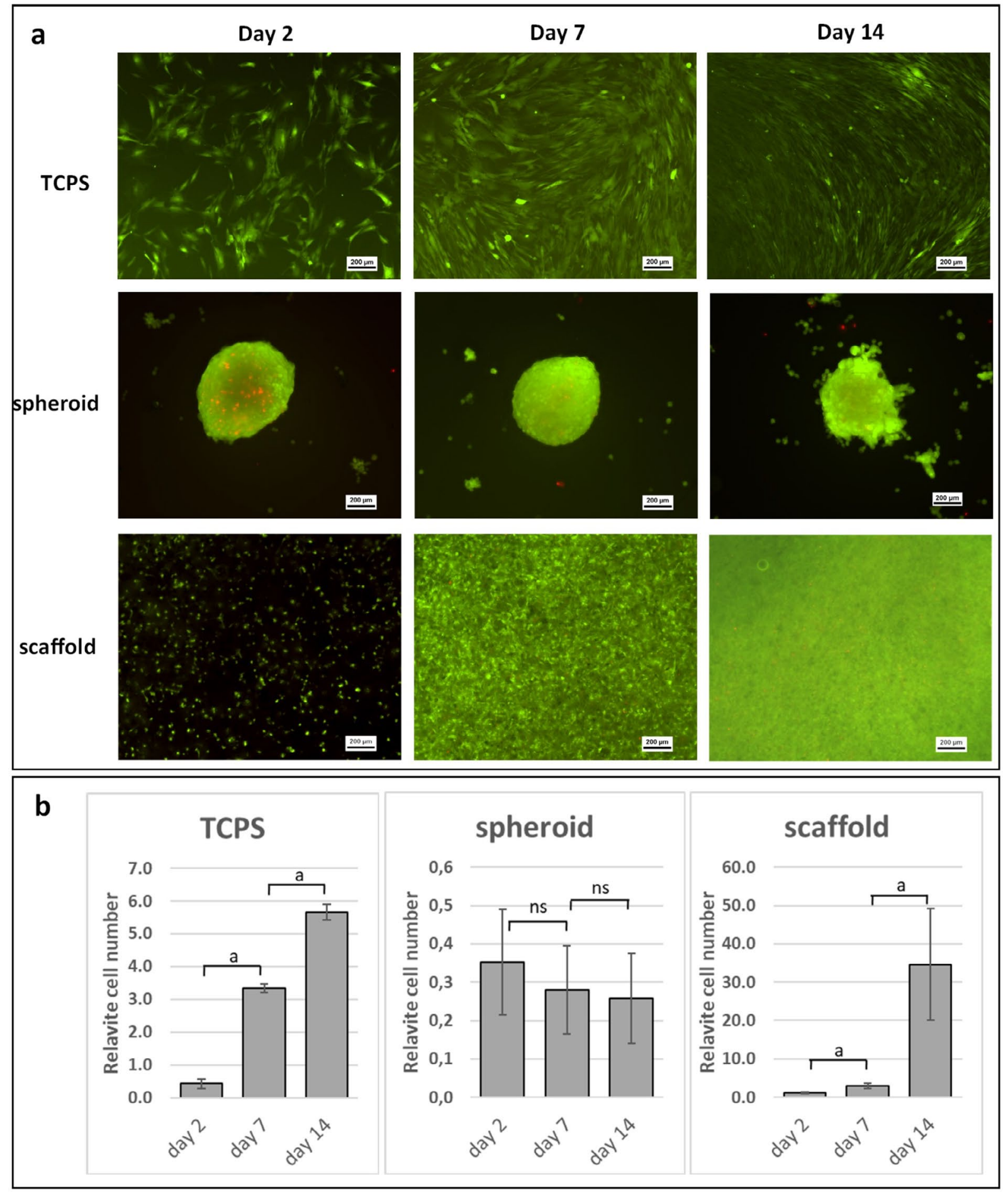

Fig. 1. Viability and proliferation of ADSCs cultured on standard TCPS, spheroids and polystyrene scaffolds. (a) Live/dead fluorescence staining of ADSC cultures - green indicates viable cells, red dead cells. Representative images of 3 experiments are shown. (b) Relative cell number of ADSCs at day 2, 7 and 14 of culture. The relative cell number was calculated based on the DNA content in the cell lysates and normalised to the value obtained for the initially seeded cell population. The presented values are the mean from 3 experiments performed on cells obtained from 3 donors; error bars show the standard deviation of the population (4 replicates per experiment, $n=12$ ). ${ }^{a} p<0.001 ;{ }^{n s} p>0.05$ in ANOVA with Tukey post-hoc test. 
Specimens were further stained for BSP, as described below. Spheroids were resuspended in mounting medium and slowly dispensed on to silanised glass slides (Dako). Scaffolds were transferred on to glass slides with fine tweezers. The cell layer formed on 24-well TCPS plates was scraped off and transferred on to the glass sides. All specimens were mounted on to the glass sides by using Fluoromount ${ }^{\mathrm{TM}}$ medium (Sigma-Aldrich). Fluorescence images of the samples were acquired with an LSM710 laser-scanning confocal microscope (Zeiss). Experiments were performed on cells isolated from three or four donors, as specified in the corresponding figure legend.

Immunofluorescence staining for BSP and osteocalcin BSP staining was performed on ADSCs cultured in 3D spheroids, 3D scaffolds and 2D TCPS after $14 \mathrm{~d}$ of osteogenic differentiation. Osteocalcin staining was performed only in 2D TCPS cultures at day 14 and 21 in osteogenic maturation experiments as described below.

Fixed cells were incubated with $10 \%$ normal goat serum containing $0.1 \%$ Triton- $X$ for simultaneous blocking and permeabilisation. After washing with PBS, cells were incubated overnight at $4{ }^{\circ} \mathrm{C}$ with primary antibody. The antibodies used were either rabbit polyclonal anti-IBSP (Sigma-Aldrich) or mouse monoclonal anti-osteocalcin (R\&D Systems). Samples were further stained with secondary antibodies: AlexaFluor $^{\mathrm{TM}} 555$ goat anti-rabbit or Cy5 goat antimouse (both ThermoFisher Scientific). The samples were analysed either with a laser-scanning confocal microscope, as described above, or with the Nikon Eclipse-Ti inverted microscope. Experiments were performed on cells isolated from three donors.

\section{Adipogenic differentiation}

ADSCs were seeded into 6-well standard culture plates (TCPS) or spheroids. Adipogenic medium (AM) was composed of culture medium supplemented with $500 \mathrm{nM}$ dexamethasone, $5 \mathrm{mM}$ indomethacin and $500 \mathrm{nM}$ 3-isobutyl-1-methylxanthine (IBMX). Cells were cultured in AM for 2 or $7 \mathrm{~d}$ and, then, lysed for RNA isolation and gene expression analysis, using the methods described above. Experiments were performed on cells isolated from three donors.

\section{Spheroid pre-differentiation and osteogenic maturation}

ADSCs were seeded to form spheroids (spheroid pre-differentiation) and on standard 6-well plates (TCPS pre-differentiation) as described above. Cells were cultured in OM for $7 \mathrm{~d}$. Next, spheroids were collected, washed with PBS and incubated for $15 \mathrm{~min}$ with Accutase solution (Sigma-Aldrich) for spheroid dissociation. Similarly, cells cultured on TCPS were detached. Accutase solution was diluted with culture medium and cells were pelleted and resuspended in OM-D3 medium. Then, cells were seeded into 24-well culture plates for osteogenic maturation. After another 7 or $14 \mathrm{~d}$ (14 or $21 \mathrm{~d}$ of culture in total), the first set of cultures was fixed for bone mineral staining and osteocalcin staining, as described above. Fluorescence of the stained bone minerals and counterstained nuclei was measured with the FLUOstar plate reader at 488/520 nm and 355/460 nm (excitation/emission), respectively. Relative content of bone minerals was expressed as relative fluorescence of the dye normalised to the nuclear fluorescence (Hoechst 33342). Microscopic images of the stained samples were acquired using the Nikon Eclipse-Ti inverted microscope. The second set of samples was lysed for RNA isolation and gene expression analysis. Undifferentiated ADSCs, cultured in CM for $2 \mathrm{~d}$, were used as negative control. Experiments were performed on cells isolated from three or four donors, as specified in the corresponding figure legend.

\section{Statistical analysis}

Each experiment was performed on cells isolated from a different donor. Each analysis in the experiment consisted of at least 3 replicates. All experiments were performed at least three times. Quantitative data from all experiments were analysed with STATISTICA software (StatSoft Inc., Tulsa, OK, USA), while the graphs were prepared using Microsoft Excel. Presented values represent the mean from all three experiments or the mean from the replicates for each experiment separately. Error bars in the graphs indicate standard deviation or $\pm 95 \%$ confidence interval of the mean. ANOVA with post-hoc Tukey test was used to assess statistical significance between the experimental groups. In gene expression experiments, $\log 2$ of the relative transcript quantity was used for statistical tests.

\section{Results}

\section{Viability and proliferation}

Efficient 3D culture systems must provide a cytocompatible environment. Therefore, viability and proliferation of ADSCs cultured in osteogenic differentiation medium in standard 24-well plates (TCPS), in spheroids and on polystyrene scaffolds were examined. Microscopic observation of fluorescently stained cultures revealed a large number of viable cells in TCPS and polystyrene scaffold cultures. With time, the available TCPS and scaffold culture surfaces were gradually colonised by viable cells. At the same time, the number of dead cells was small. In spheroid cultures, most cells that formed spheroids were viable; however, dead cells were observed in the core of the spheroid (Fig. 1a). The relative number of cells in the investigated cultures was assessed with the PicoGreen DNA quantification assay performed on cell lysates. TCPS and polystyrene scaffold cultures showed increasing DNA content during culture, indicating that cell proliferation occurred. The mean fold increase in DNA content at day 14 as compared to day 0 in TCPS and polystyrene scaffold cultures was 5.7 and 34.6, respectively. The larger relative 


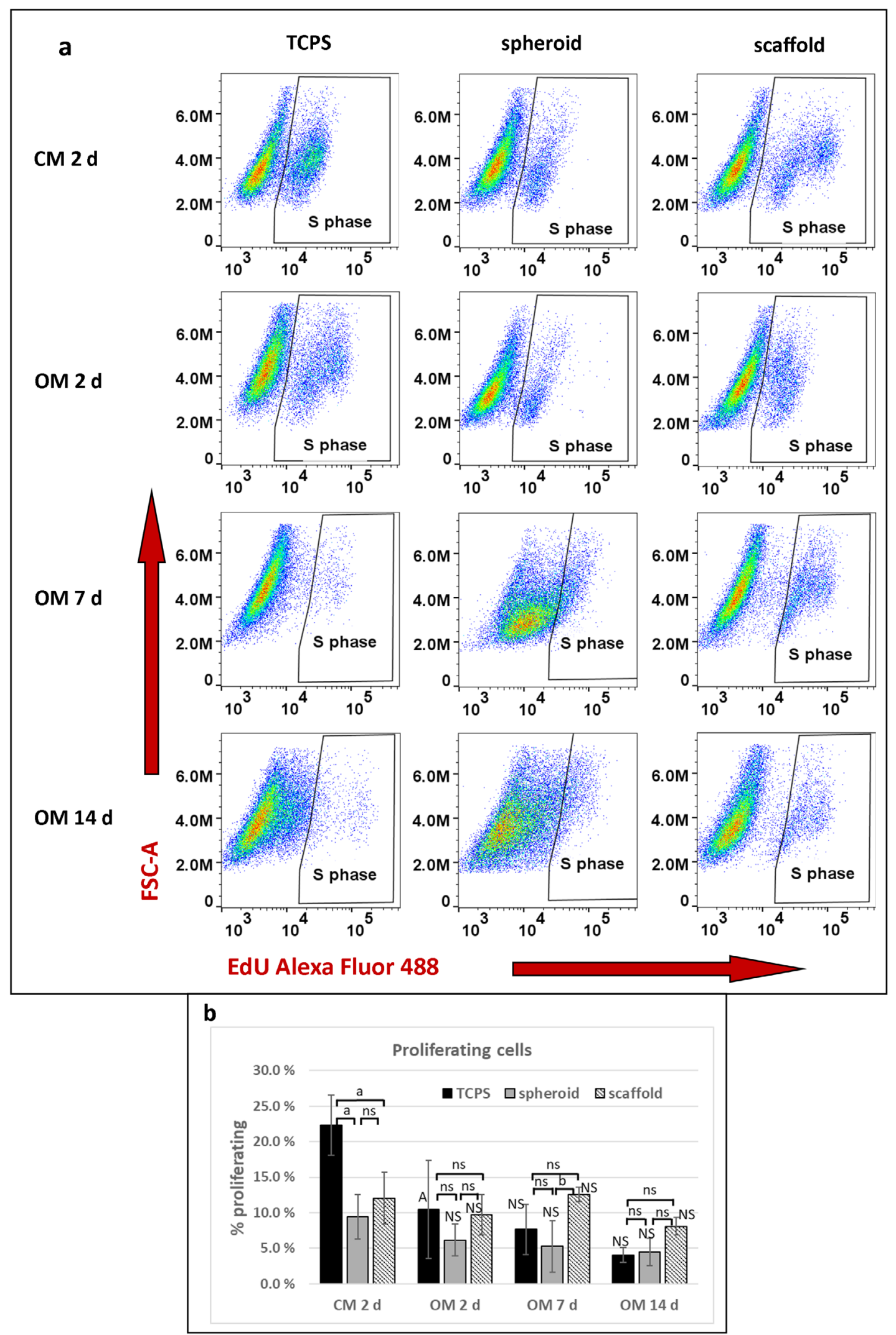

Fig. 2. The effect of 3D culture on ADSCs cell proliferation. ADSCs were cultured for $2 \mathrm{~d}$ in $\mathrm{CM}$ and OM in 2D TCPS, spheroids or scaffolds. Additionally, OM cultures were analysed also at day 7 and 14. Cells were pulsed for $2 \mathrm{~h}$ with EdU before staining and flow cytometric data acquisition. (a) Representative dot plots show S-phase populations. (b) Mean population frequencies of cells in S-phase are plotted on the chart. Error bars represent standard deviation $(n=4)$. Lower-case letters mark the statistical significance between the samples in one medium-time point condition, while capital letters show significance between adjacent conditions in one culture type (e.g. A marks statistical significance between the TCPS samples in $\mathrm{CM} 2 \mathrm{~d}$ group and OM $2 \mathrm{~d}$ group). ${ }^{\mathrm{a}} p<0.001{ }^{\mathrm{b}} \mathrm{b}=0.013$; ns: not significant; ANOVA with Tukey post-hoc test. 
number of cells in the scaffold cultures at day 14 might have been caused by the larger available cell culture surface area as compared to that of the TCPS cultures. On the other hand, the relative number of cells in spheroids did not change significantly with time in culture (Fig. 1b). Therefore, the number of proliferating cells was evaluated in all cultures with a flow cytometer and EdU-pulsed cell samples. Mean relative number of proliferating cells in $2 \mathrm{D}$ TCPS cultured in CM with FGF-2 was significantly larger than in 3D spheroids and 3D scaffolds. However, cell proliferation in 2D TCPS was significantly downregulated when $\mathrm{OM}$ was used instead of $\mathrm{CM}$ with FGF-2. Cell proliferation in 3D spheroid and 3D scaffold was not affected by switching the cells into OM. Differences in cell proliferation among different culture systems in OM did not significantly differ, with one exception at day 7 , where the proliferation rate on 3D scaffolds was significantly higher than in 3D spheroids (Fig. 2).

\section{ALP activity}

To determine the effect of the 3D systems on early osteogenic commitment, ALP activity in ADSCs was analysed at days 2 and 7 of culture in osteogenic differentiation medium (Fig. 3). ALP activity peaked at day 2 in spheroid cultures and was significantly higher as compared to that in TCPS and polystyrene scaffold cultures. At day 7, ALP activity in spheroids dropped to the level of the undifferentiated control. On the other hand, cells cultured on polystyrene scaffolds had significantly elevated ALP activity as compared to that of all other experimental groups at day 7. ALP activity in TCPS-cultured cells remained unaltered through the entire experiment, compared to that of the undifferentiated control cells (Fig. 3). Spheroid cultures provided efficient up-regulation of the early differentiation marker as compared to
2D TCPS. Polystyrene PS scaffolds also proved to be beneficial for early osteogenic differentiation, but the regulation was evident at a later time point. ALP activity up-regulation was not observed in 2D TCPS cultures, which might be due to the limited culture time of the experiment.

\section{Osteogenic differentiation molecular markers}

The transcriptional activity of RUNX2 and osterix drives the osteogenic differentiation of MSCs. Therefore, the expression of genes encoding these transcriptional factors was analysed in the tested experimental conditions, during $14 \mathrm{~d}$ of culture. RUNX2 expression was significantly elevated in spheroids as compared to the other tested conditions at day 2. Polystyrene scaffold culture did not have a significant effect on the mean RUNX2 expression, compared to 2D TCPS. However, the mean RUNX2 expression in scaffolds was lower than in spheroids at day 2. At day 14, RUNX2 expression in the given culture system was similar to day 7 and 2. An elevated level of RUNX2 in spheroids at day 2 was also confirmed at the protein level by Western blot analysis (Fig. 4b).

Osterix gene expression followed a similar pattern. The transcript level was 38.9-fold higher in spheroid cultures at day 2 and was the highest among all tested conditions. The mean gene expression was significantly higher in spheroids than in 2D TCPS, but the difference between spheroids and scaffolds did not reach statistical significance due to high inter-donor variability. Nonetheless, intra-donor comparison of osterix gene expression showed lower transcript levels in scaffolds as compared to spheroids, for each of the three cell donors tested. Expression levels in cells cultured on polystyrene scaffolds were also not significantly different from the ones observed in spheroids (Fig. 4a). Gene expression

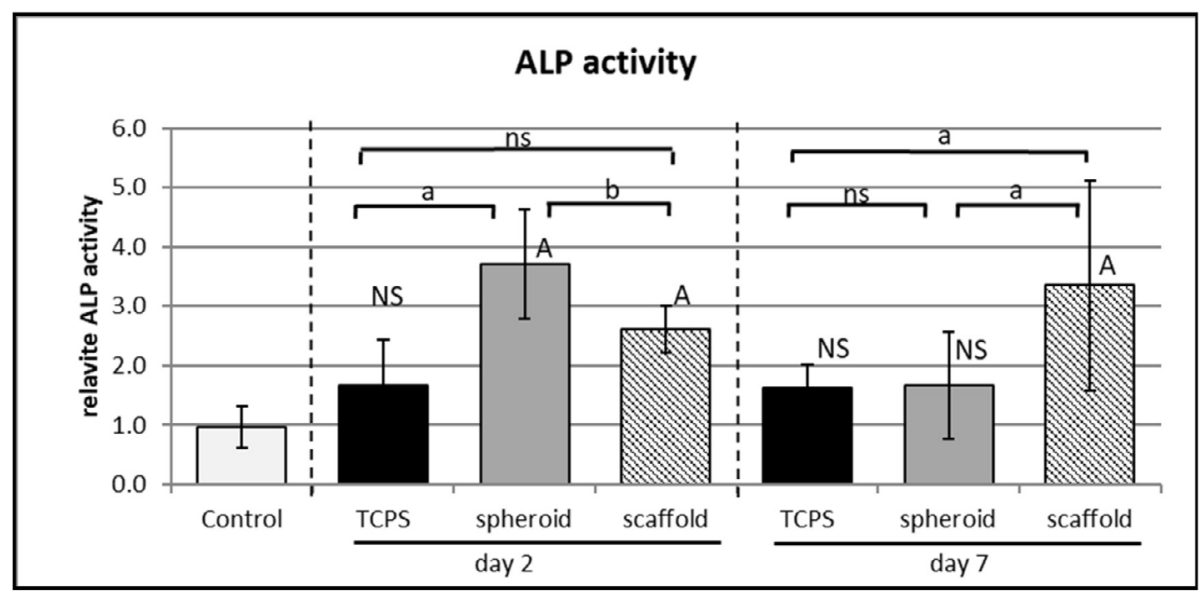

Fig. 3. ALP activity in ADSCs cultured on standard TCPS, spheroids and polystyrene scaffolds. Values obtained in each experiment were normalised to the control cell culture - undifferentiated cells cultured on standard 6-well plates at day 2 (TCPS). Graph shows mean relative ALP activity. Lower-case letters mark the statistical significance between the samples in one medium-time point condition, while capital letters show significance of sample to control comparison. The experiments were performed on cells obtained form 3 donors. Error bars represent standard deviation $(n=12) .{ }^{a} p<0.001 ;{ }^{b} p=0.0414$; ${ }^{\text {ns }} p>0.05$; ANOVA with Tukey post-hoc test. 
decreased at day 7 and 14 as compared to day 2 in spheroid cultures. In scaffold and TCPS cultures at day 7 and 14, the level of osterix gene was similar to that of undifferentiated control cells.

Genes encoding selected bone matrix proteins, such as COL1A1 and IBSP, were also analysed. The expression of COL1A1 was the highest at day 2 in all three culture systems and the transcript level decreased with culture time, up to day 14 . The differences between the two 3D systems (spheroids and polystyrene scaffolds) and 2D TCPS were not statistically significant. However, the expression of IBSP was substantially regulated by the $3 \mathrm{D}$ spheroid culture. The highest mean expression level was
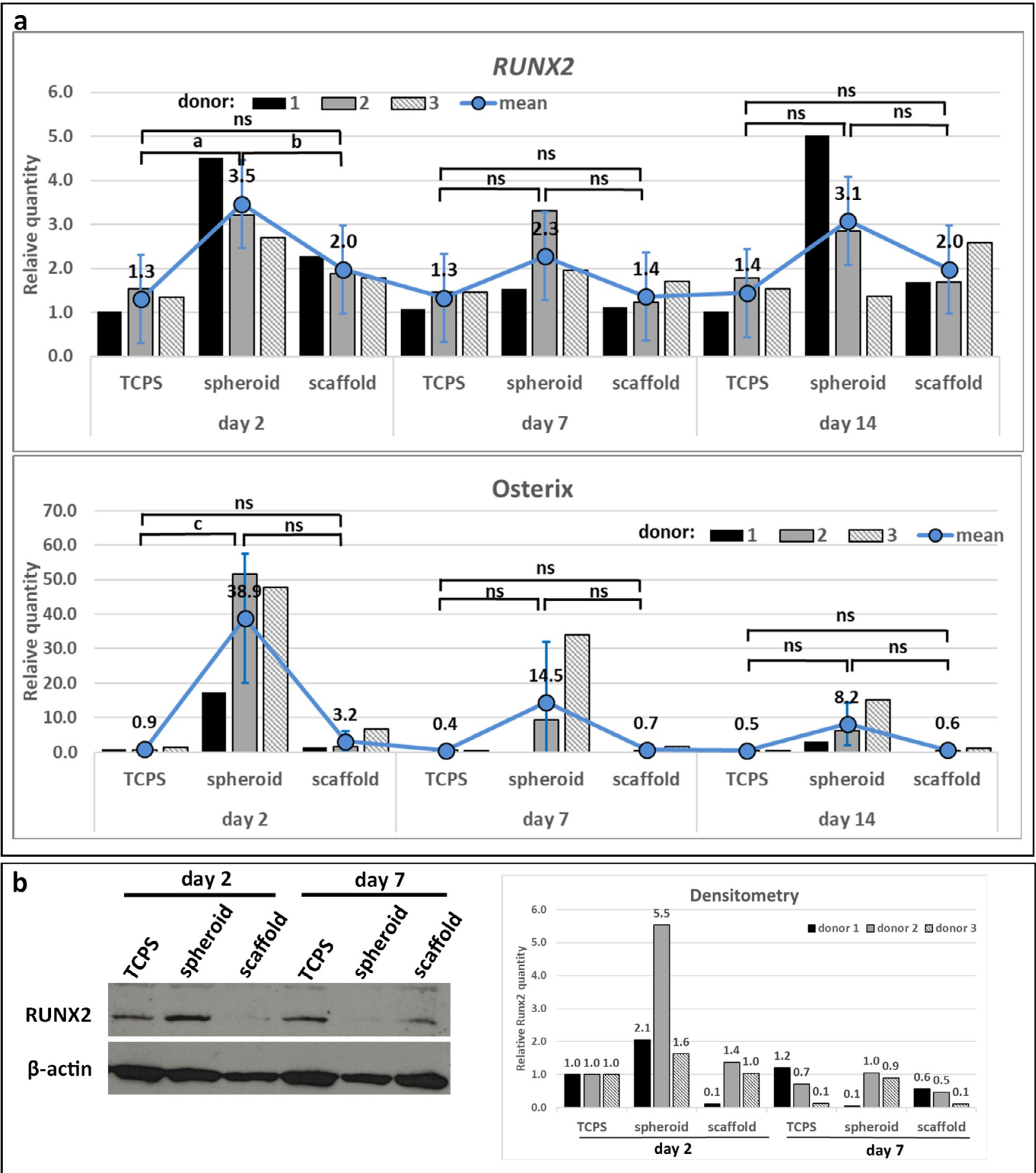

Fig. 4. RUNX2 and osterix gene expression in ADSCs cultured on standard TCPS, spheroids and polystyrene scaffolds. (a) Quantitative reverse transcription PCR (qRT-PCR) for RUNX2 and osterix gene - values represent the relative transcript quantity in each of the cell donors tested and mean from three experiments, where each of the experiments was performed on cells isolated from a different donor. Error bars represent standard deviation. ${ }^{\mathrm{a}} p=0.0343 ;{ }^{\mathrm{b}} p=0.0429 ;{ }^{\mathrm{c}} p=0.0155 ;{ }^{\mathrm{ns}} p>0.05$; ANOVA with Tukey posthoc test. (b) Representative Western blot for RUNX2 protein and densitometric analysis of all the performed blots. $\beta$-actin was used as a loading control. The experiment was performed 3 times on cells isolated from different donors. 
observed at day 7 in spheroids and reached 824-fold as compared to the level in the reference sample. In 2D TCPS cultures and polystyrene scaffolds, the values ranged between 3.8- and 0.1-fold and were not significantly different from the reference sample (Fig. 5). At day 14, the level of IBSP in spheroids was downregulated as compared to day 7 time point. The mean IBSP expression level in spheroids was significantly upregulated as compared to 3D TCPS and scaffolds at all three time points tested. The obtained results indicated that spheroids upregulated the expression of three out of four tested osteogenesis molecular markers.

Production of bone extracellular matrix components Production of bone extracellular matrix components is a hallmark of intermediate and late differentiation events. In the present study, BSP and bone mineral deposition were fluorescently stained in $14 \mathrm{~d}$ cultures and images of the prepared samples were acquired using a confocal microscope. BSP was produced only by ADSCs in spheroids and not in polystyrene scaffolds or monolayer cultures (Fig. 6a). On the other hand, bone mineral production was efficient in monolayer and polystyrene scaffolds, but the stained mineral area was low in spheroid cultures (Fig. 6b). Therefore, spheroid cultures enabled production of $\mathrm{BSP}$, one of the characteristic bone matrix proteins. However, bone mineral production was inhibited in spheroids as compared to the other two culture systems. The low mineral level in spheroid cultures might have been caused by inhibition of matrix mineralisation, originating from delayed osteoblast maturation. Alternatively, the spheroid environment might prevent stable association of the cell-derived hydroxyapatite (HA) in cell culture due to lack of external adhesion surface, i.e. TCPS, which is present in monolayer and scaffold cultures and might contribute to HA binding. On the other hand, 2D TCPS and scaffold cultures did not produce BSP, one of the proteins crucial for binding of HA in the bone extracellular matrix. Therefore, the described results did not give a definite answer regarding the efficiency of osteogenic maturation in the testes culture systems.

\section{Adipocyte differentiation in spheroids and on 2D TCPS}

The results of the osteogenic differentiation experiments performed in the three tested systems indicated that spheroids provided a favourable
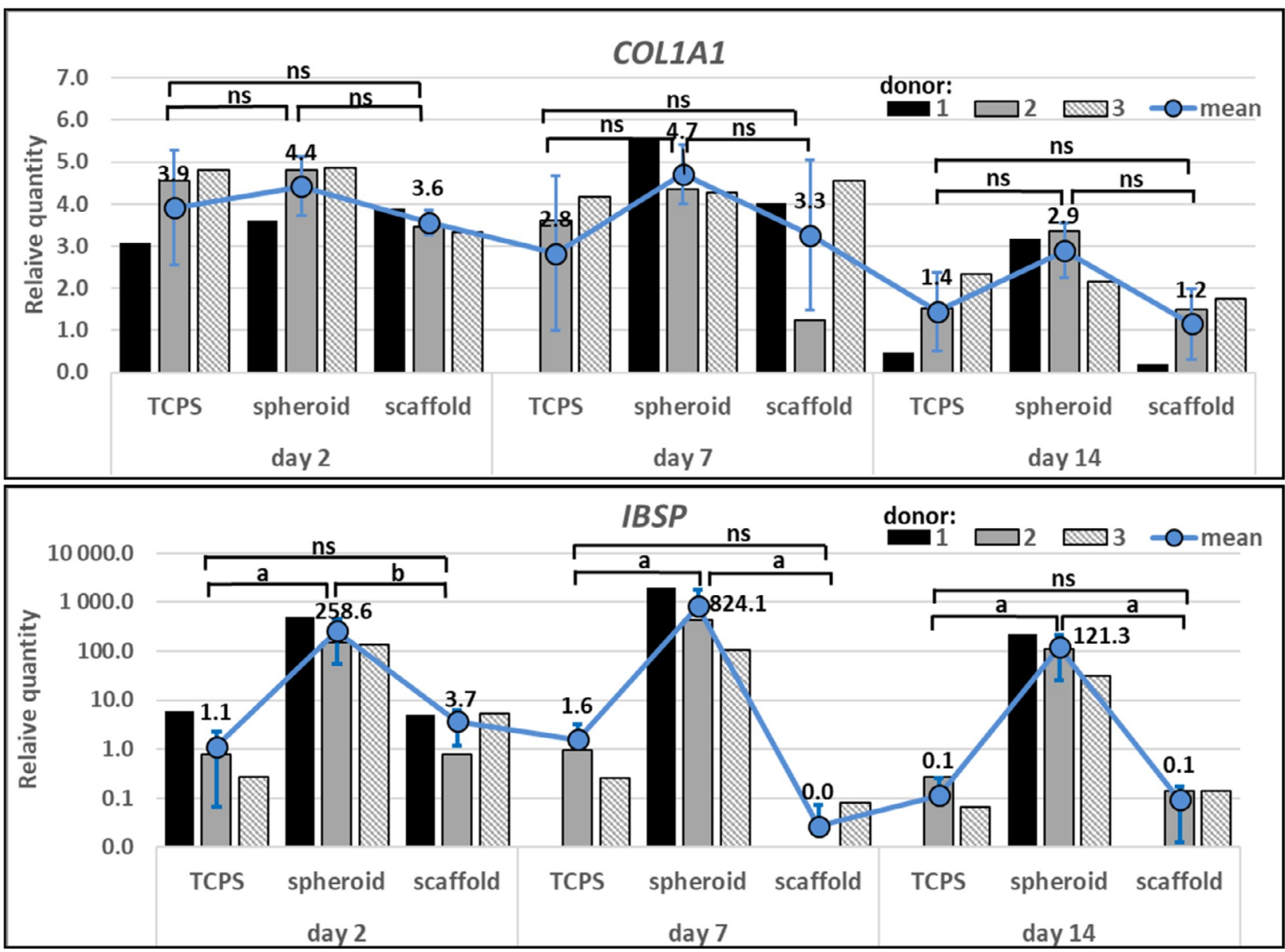

Fig. 5. COL1A1 and IBSP expression in ADSCs cultured on standard TCPS, spheroids and polystyrene scaffolds. Values represent the relative transcript quantity in each of the cell donors tested and the mean from three experiments, where each of the experiments was performed on cells isolated from a different donor. Error bars represent standard deviation. ${ }^{\mathrm{a}} p<0.001 ;{ }^{\mathrm{b}} p=0.0051 ;{ }^{\mathrm{ns}} p>0.05$; ANOVA with Tukey post-hoc test. 
environment for osteogenic commitment. Hence, the working hypothesis was that the increased expression of early and intermediate markers of osteogenesis in ADSC spheroids might originate from the inhibited adipogenic differentiation capacity in spheroids. The expression of early and intermediate osteogenesis markers was only moderately affected in cells cultured on polystyrene scaffolds; therefore, the scaffolds were not included in the adipogenesis experiments. To test this hypothesis, ADSCs were seeded into 2D TCPS and spheroids for $7 \mathrm{~d}$ culture in AM. Both types of cultures responded to AM with up-regulated expression of the four tested genes. The differences in mean expression of PPARG, CEBPA, FABP4 and LPL between 2D TCPS and spheroids at both time points were not significant. However, CEBPA, FABP4 and LPL expression levels were lower in spheroids as compared to 2D TCPS, when analysing the results for each of the cell donor separately. The high inter-donor variability might have hampered reaching statistical significance between the experimental groups (Fig. 7). Therefore, the obtained results did not support the hypothesis that the spheroids culture could affect adipogenic differentiation of ADSCs. Further studies, using a larger group of cell donors, could be useful in elucidating if the observed trend of decreased CEBPA,
FABP4 and LPL expression in spheroids would reach statistical significance.

Impact of osteogenic pre-differentiation in spheroids on late osteogenic events in 2D

Data obtained on BSP and bone mineral staining suggested that bone extracellular matrix deposition, a process characteristic of intermediate and mature osteoblasts, occurs differently in spheroids than in attachment-permissible systems (TCPS and polystyrene scaffolds). Specifically, bone mineral deposition was found to be less efficient in spheroids than in the remaining two systems. Spheroids might provide a less favourable environment for mineral deposition than ADSCs cultured on polystyrene. Therefore, the question whether the spheroidderived, pre-differentiated ADSCs, when plated back into 2D TCPS culture, would produce bone minerals efficiently was studied. Polystyrene scaffolds were not included in these experiments as bone mineral production was at a similar level as in 2D TCPS culture. Therefore, ADSCs were pre-differentiated for $7 \mathrm{~d}$ in either spheroids or 2D TCPS and, then, cultured in osteogenic maturation medium for an additional 7 or $14 \mathrm{~d}$ in 2D TCPS. This experimental procedure allowed the study of osteogenic maturation beyond the $14 \mathrm{~d}$ time point, which would be otherwise

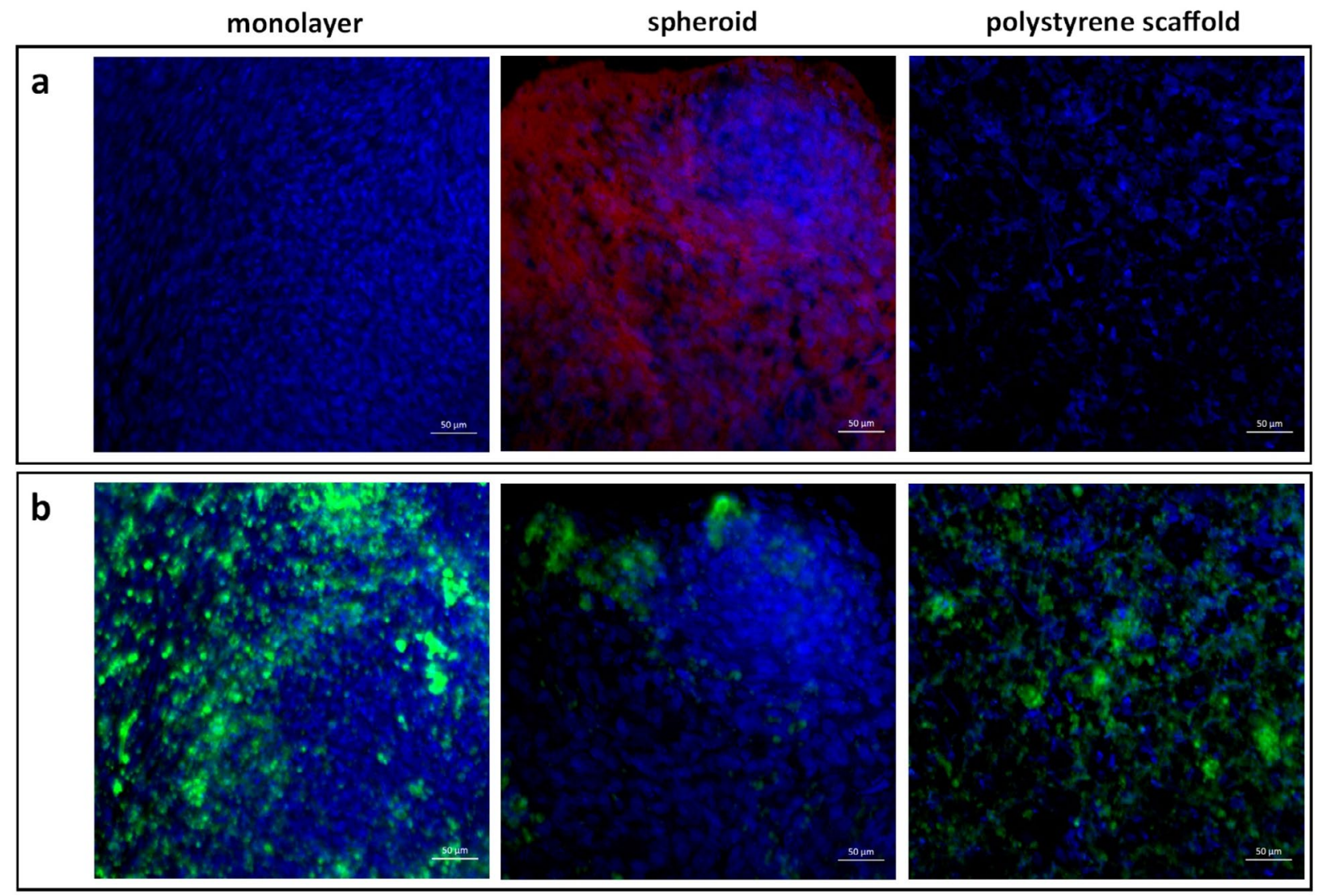

Fig. 6. Bone mineral and BSP staining at day 14 in ADSCs cultured on standard TCPS, spheroids and polystyrene scaffolds. BSP was detected with Alexa Fluor ${ }^{\mathrm{TM}} 555$ secondary antibody (red colour); bone mineral deposition was detected with the OsteoImage ${ }^{\mathrm{TM}}$ assay (Lonza) (green colour). The panels shows (a) BSP or (b) bone mineral staining merged with nuclear counterstaining (blue colour). The images show representative results obtained from 3 experiments, performed on cells obtained from 3 donors. Scale bars: 50 $\mu \mathrm{m}$. 
impossible to achieve in continuous spheroid culture (spheroids culture could not be maintained up to day 21). Additionally, possible effect of enhanced osteogenic induction in spheroids on osteoblast maturation could be determined in the applied approach. Spheroid pre-differentiation and dissociation protocol would allow taking the advantages of both culture methods: enhanced early osteogenesis in spheroids and efficient mineralisation in 2D TCPS.

Bone mineral staining performed after a total of $21 \mathrm{~d}$ showed evident mineral production in the investigated samples. Not surprisingly, at day 14 , mineral deposition was not detected in either of the culture conditions (Fig. 8a). The fluorescence intensity emitted by the mineral staining reagent was further quantified using a multi-well plate reader to compare the amount of mineral deposited in pre-spheroid and pre-TCPS cultures at day 21 . No differences in relative fluorescence intensity were observed, indicating that the efficiency of mineral deposition was not affected by the pre-differentiation method (Fig. 8b). Considering that osteocalcin is a well-known marker of mature osteoblasts (Capulli et al., 2014; Liu and Lee, 2013), osteocalcin was studied at the gene and protein levels in the investigated experimental setup. Expression of osteocalcin gene was higher in the spheroid pre-differentiated cell population than in 2D TCPS pre-differentiated cells, despite not being statistically significant (Fig. 9a). Interestingly, the relative gene expression was higher at day 14 than at day 21 in both culture systems. At the same time, osterix gene expression did not increase above the level observed in the reference sample. Osteocalcin protein was evaluated with immunofluorescence staining in both spheroid and TCPS pre-differentiated cells at day 14 and 21. Osteocalcin was detected in both culture systems at day 14 . Consistently with the qRT-PCR results, the staining intensity at day 21 decreased in both pre-differentiation study groups as compared to day 21 (Fig. 9b).

\section{Discussion}

The present work studied the effect of 3D culture microenvironments on the osteogenic differentiation of ADSCs. Several studies have already shown the osteoinductive effect of 3D biomaterial scaffold (Gabbay et al., 2006; Lee et al., 2011; Rumiński et al., 2018; Xue et al., 2017) and chitosan-derived spheroids (Cheng et al., 2012; Hsu and Huang, 2013) on ADSCs in vitro. The current study aimed to exclude additional experimental variables, such as integrin activation by culture surface components and signaling by released ions or growth factors, that may affect the differentiation of ADSCs. The 3D systems utilised in the study were designed to not
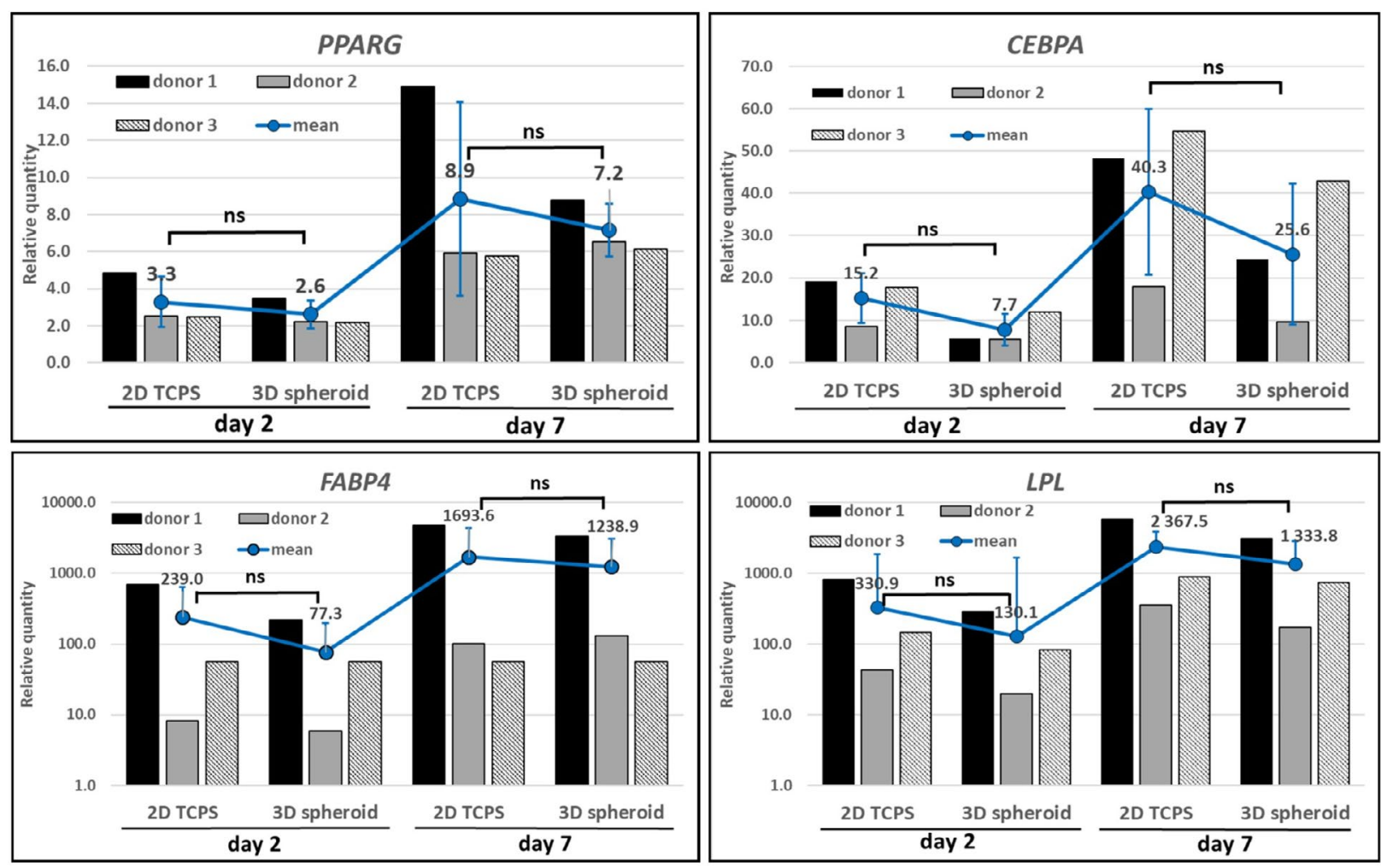

Fig. 7. Adipocyte differentiation of ADSCs cultured on standard TCPS, spheroids and polystyrene scaffolds - expression of PPARG, CEBPA, FABP4 and LPL at day 2 and 7. Values represent the relative gene expression in each of the cell donors tested and the mean from three experiments, where each of the experiments was performed on cells isolated from a different donor. Error bars represent standard deviation. ${ }^{n s} p>0.05$; ANOVA with Tukey post-hoc test. 
apply any external biochemical or physical stimuli to the cells - the applied scaffold was manufactured from polystyrene, which is a routinely used material for standard culture dishes, while the spheroids did not contain any attachment-supportive material.

All three studied culture conditions provided a cytocompatible culture environment. The relative number of cells in the 2D TCPS and polystyrene scaffold cultures increased with time in culture, indicating that cells proliferated effectively. On the other hand, the relative number of cells in spheroids did not change with time. Analysis of the DNAreplicating cell population in spheroids proved that the spheroid environment negatively regulated cell proliferation in CM. Similarly, other studies have shown the presence of BrdU- or Ki67-positive BMSCs in spheroids, with proliferating cells located at the periphery of the spheroids (Baraniak and McDevitt, 2012; Burns et al., 2010). No change in the relative number of cells in spheroids was observed, which might be attributed to cell death or to the spontaneous disassociation of spheroids. Finally, switching culture medium in 2D TCPS culture to OM resulted in downregulation of proliferation, which is likely linked with the onset of differentiation.

Analysis of markers of the early and middle osteogenic differentiation stages revealed substantial differences among the studied culture systems. The enzymatic activity of ALP, an early marker of osteogenesis, was higher in polystyrene-scaffoldcultured cells than in 2D TCPS at day 7. In the case of spheroid cultures, up-regulated ALP activity was reported even earlier, at day 2. Furthermore, in terms of marker gene expression, the most efficient differentiation was observed in ADSC spheroids, followed by polystyrene scaffold cultures and 2D TCPS. Interestingly, the most striking difference between spheroids and the other two systems was observed for osterix and IBSP, whose transcript levels were up-regulated by approximately 1 and 2 orders of magnitude, respectively.

Up-regulated expression of osteogenesisassociated genes in human BMSC spheroids was previously reported by several other authors. Wang and co-authors (2009) performed qRT-PCR on monolayer culture and spheroids and reported up-regulated expression of RUNX2 in spheroids. Furthermore, an oligonucleotide array experiment proved that other osteogenesis-related genes, including $A L P, B M P 2$ and $S P P 1$, are up-regulated in BMSC spheroids (Wang et al., 2009). Kabiri et al. (2012) reported up-regulated expression of RUNX2, BMP-2, osteonectin and collagen I genes in spheroids as compared to monolayer culture. Ho et al. $(2017,2016)$ reported enhanced ALP activity and osteocalcin secretion in alginate-entrapped spheroids as compared to monolayer BMSCs. On the other hand, ALP activity remains unaltered while calcium content is up-regulated in spheroids as compared to monolayer cells at day 7 and 14 of osteogenic differentiation (Kabiri et al., 2012), while differences in ALP activity and mineralisation between spheroid and monolayer BMSCs are reported (Murphy et al., 2014). Interestingly, Murphy et al. (2016) reported down-regulated ALP activity in spheroids after $12 \mathrm{~d}$ of osteogenic culture. In contrast to BMSC studies,

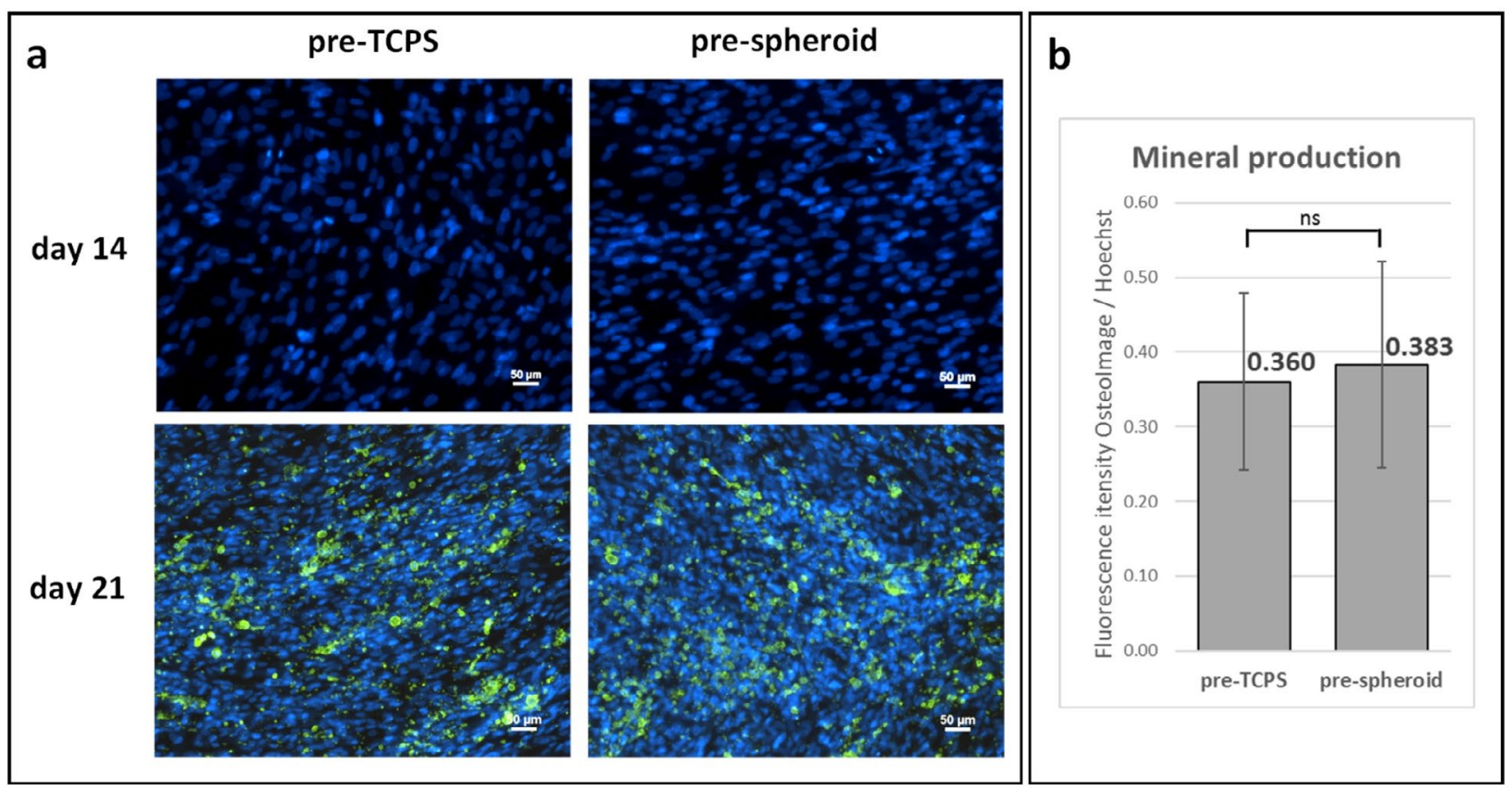

Fig. 8. Effect of spheroid pre-differentiation on osteoblast maturation - mineral production. (a) Representative fluorescence microscopy images of cultures stained for bone minerals (green colour) and nuclei (blue colour). (b) Fluorescence intensity measurement of stained samples in a multi-well plate fluorimeter. Graph shows mean fluorescence intensity of the mineral stain measured in four experiments, performed on cells obtained from 4 donors ( 4 replicates per experiment, $n=16$ ). Values were normalised to Hoechst 33342 blue fluorescence to correct for the cell number in the sample. ns: not significant. Scale bars: $50 \mu \mathrm{m}$. 
data regarding the osteogenesis of ADSC spheroids are relatively scarce. Up-regulation of RUNX2 in human ADSCs is observed in chitosan-generated spheroids as compared to 2D cultures (Cheng et al., 2012). Furthermore, Yamaguchi et al. (2014) studied the osteogenic differentiation of rat ADSCs cultured on low-binding plates. Similarly to the present study, gene expression of RUNX2, BSP, OPN and osterix was up-regulated in ADSC spheroids.
The various techniques used for spheroid fabrication and the different parameters evaluated to assess osteogenesis efficiency produce some inconsistencies among studies but, as a general conclusion, osteogenic differentiation is either enhanced or not affected by the spheroid culture conditions. Therefore, the present study results were in agreement with the literature data presented above. In addition, the study data might provide
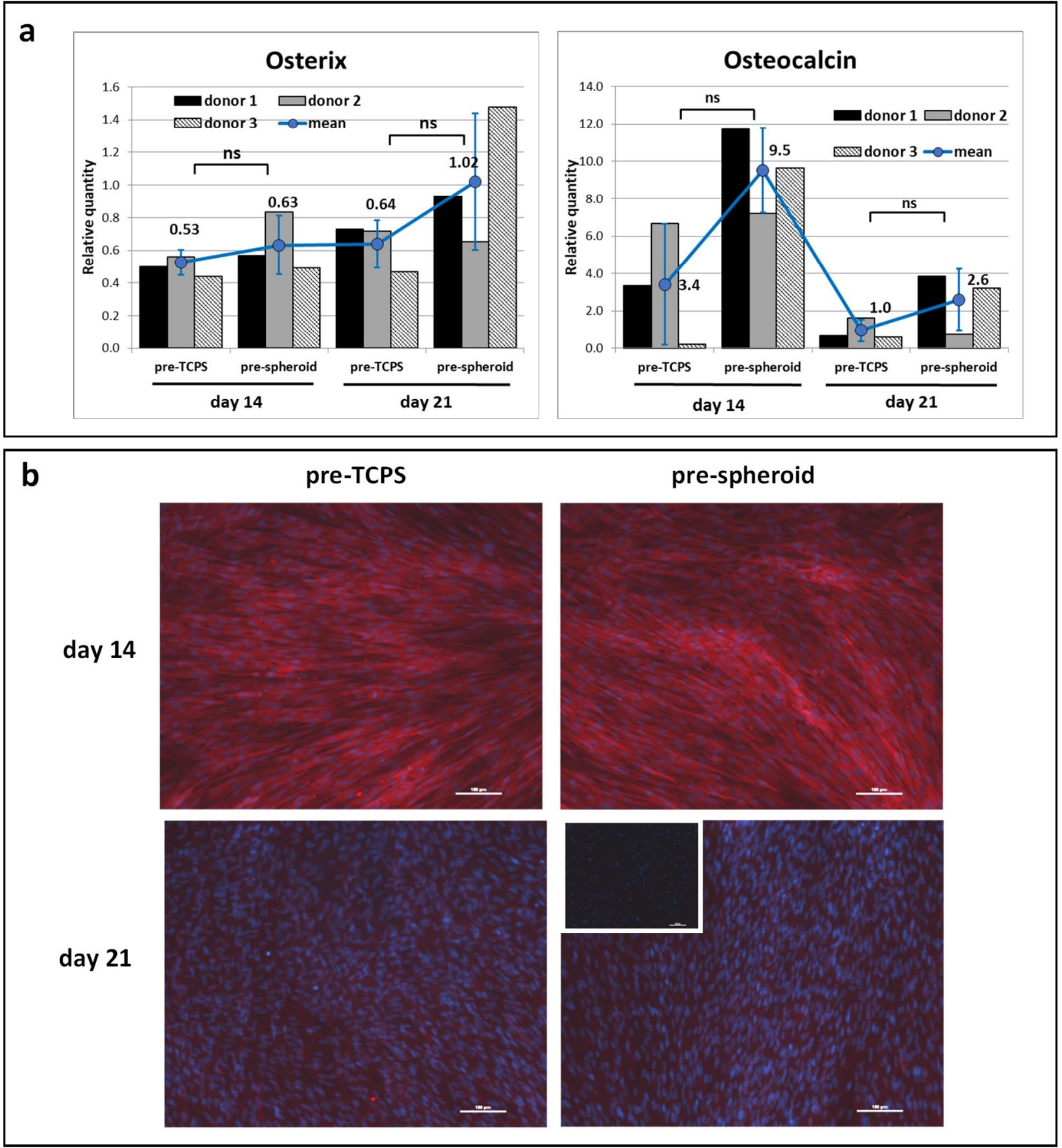

Fig. 9. Effect of spheroid pre-differentiation on osteoblast maturation - osteocalcin detection. (a) qRTPCR for osteocalcin and osterix mRNA - values represent the relative quantity in each of the cell donors tested and the mean from three experiments, where each experiment was performed on cells isolated from a different donor. Error bars represent standard deviation. ${ }^{n s} p>0.05$; ANOVA with Tukey post-hoc test. (b) Representative fluorescence microscopy images of cultures stained for osteocalcin protein (red colour, secondary reagent - a Cy5-conjugated antibody) and nuclei (blue colour). The experiment was performed 3 times on cells isolated from 3 donors. The insert in the bottom left picture shows undifferentiated ADSCs stained for osteocalcin - negative control. Scale bars: $100 \mu \mathrm{m}$. 
several interesting insights regarding osteogenic differentiation, which might be characteristic of ADSCs. First, spheroid culture was found to be an efficient method to enhance ALP activity and might, at least partially, compensate for the intrinsically low activity of this enzyme in ADSCs (Kalaszczynska et al., 2013). Second, the most prominent regulation was observed for osterix and IBSP genes, while moderate regulation was observed for RUNX2 and collagen type I gene. As the first two markers are found predominantly in osteoblasts and the last two are characteristic of pre-osteoblasts (Liu and Lee, 2013), the spheroid culture seemed to provide an efficient switch between early and middle differentiation events, which otherwise does not occur in 2D culture. However, curiously, maturation of the bone extracellular matrix was inhibited by spheroid conditions. Finally, interesting differences were observed in the production of bone matrix components - BSP and bone minerals. In agreement with the qRT-PCR results, BSP was detected only in spheroid cultures. On the other hand, bone mineral production was lower in spheroid cultures than in 2D TCPS and polystyrene scaffold cultures. This suggests that osteogenic commitment and bone extracellular matrix production occur differently in a system without an exogenous attachment surface than in TCPS-supported adherent cultures. Most of the results published for BMSCs differ in that regard since mineralisation results enhanced in BMSC spheroids as compared to monolayer culture, as evidenced by calcium content analysis (Kabiri et al., 2012; Wang et al., 2009) or alizarin red staining (Wang et al., 2009). Therefore, results suggested that the spheroid environment might inhibit the osteogenic maturation of ADSCs. Considering that ADSC spheroids cultured in growth medium express higher levels of stemness-associated molecular markers (Cheng et al., 2013; Cheng et al., 2012), it seems plausible that the osteogenically differentiating spheroids might partially maintain the stem-like characteristics of ADSCs and, thus, inhibit terminal differentiation. Furthermore, ADSCs cultured in spheroids showed persistently high levels of Runx 2 mRNA up to day 14, compared to monolayer and scaffold cultures. Although expression of Runx2 directs differentiation of mesenchymal cells to the osteoblastic lineage and induces expression of bone-matrix genes, maturation of osteoblasts is actually repressed by Runx2 (Bruderer et al., 2014; Liu and Lee, 2013). Consequently, the spheroid environment might block the differentiation process at the immature osteoblast stage, which would explain inhibited bone mineral production. This hypothesis was strengthened by the observations performed after dissociation of spheroids, where low levels of osterix expression could be observed. On the other hand, low levels of osterix were accompanied by efficient mineralisation. As osterix is a downstream factor of Runx2 and its expression is positively regulated by Runx2 (Nishio et al., 2006), the expression level of osterix might be considered to be an indirect marker of Runx2 transcriptional activity. Therefore, it is plausible that spheroid dissociation might inhibit Runx2 activity, releasing a blockade for osteoblast maturation and permitting mineralisation. However, this hypothesis would have to be tested in future experiments, for example on ADSCs engineered to express high and low levels of Runx2. In addition to the analysis of standard osteogenesis markers, valuable information could be obtained by analysing transcriptional activity of Runx2 with the use of a reporter gene assay, e.g. luciferase assay, with the promoter of one of the Runx2 target genes, e.g. COL1A1 promoter, cloned upstream of the luciferase gene. As Runx2 activity is tightly regulated both at the transcriptional and protein level, using reporter gene assays would provide accurate data on the role of Runx2 activity in all stages of osteogenic differentiation.

The second 3D culture model analysed in the present study, the 3D polystyrene scaffold, also provided enhanced osteogenic commitment, but to a lesser extent than the 3D spheroids - only ALP activity was up-regulated, while RUNX2, IBSP and osterix gene expression remained unaffected. The 3D architecture of PCL-based scaffolds stimulates the osteogenesis of ADSCs (Rumiński et al., 2018; Xue et al., 2017). However, to the best of the authors' knowledge, there are currently no studies describing enhanced osteogenesis of ADSCs in a non-degradable, biologically inert 3D scaffold, such as the polystyrene applied in the present study. Comparison of the data obtained for all three culture systems suggested that the differentiation process in attachment-dependent conditions (polystyrene scaffolds and 2D TCPS) shares common characteristics that are different from those of the attachment-independent system (spheroids). As discussed above, the attachmentindependent system is unique in terms of its high osterix and BSP expression and low mineralisation. The products of both regulated genes play important roles in bone homeostasis in vivo. Osterix, apart from driving gene expression of bone extracellular matrix proteins, is indispensable for the maintenance of the long-term haematopoietic stem cell (HSC) population in the bone marrow (Coskun et al., 2014). $\mathrm{BSP}$, on the other hand, is necessary for proper bone mineralisation and the presence of enough osteoclast progenitors in vivo (Malaval et al., 2008). Therefore, steering ADSC differentiation into osterix- and BSPpositive cells might improve their function after implantation in vivo.

Interestingly, high mineral production was observed both in 3D polystyrene scaffolds and in 2D TCPS cultures, suggesting that the attachment of cells to a solid substrate might play a role in the mineralisation process. A pioneering work by Engler et al. (2006) provided evidence that MSC fate may be affected by cell culture substrate stiffness - rigid substrates induce expression of, among others, Run $x 2$ 
and osteocalcin gene, thus directing MSCs towards the osteogenic lineage. On the other hand, stiff substrates allow cells to spread, enhancing integrin binding and generating cytoskeleton tension mediated by non-muscle myosin II and inducing RhoA GTPase and Rho kinase (ROCK) activity (Steward and Kelly, 2015). Culture on polystyrene scaffolds provides a rigid environment with a Young's modulus of approximately $10^{9} \mathrm{~Pa}$, which is close to the rigidity of bones (Young's modulus: $10^{5}-10^{10} \mathrm{~Pa}$ ) (Baraniak et al., 2012; Jhala and Vasita, 2015). In vitro mineralisation, studied in MC3T3-E1 pre-osteoblast cell line, was found to be most efficient on stiff polystyrene as compared to softer polyacrylamide-collagen gels with a Young's modulus of 11-39 kPa (Khatiwala et al., 2006). Similarly, the amount of mineralisation in ADSCs cultures maintained in osteogenic medium is enhanced on stiff polydimethylsiloxane (PDMS) substrates, while a soft PDMS inhibits mineralisation and enhances adipocyte differentiation (Zhang et al., 2018). As RhoA/ROCK activation in MSCs induces osteogenic differentiation and mineral production (Strzelecka-Kiliszek et al., 2017), it is not surprising that soft culture substrates inhibit osteogenesis. Therefore, the inefficient mineralisation observed in the present study in spheroids might be the consequence of a low RhoA/ROCK pathway activity. Other research groups have fabricated BMSCs spheroids with incorporated gelatine microparticles, to increase the stiffness of spheroids (Abbasi et al., 2018; Baraniak et al., 2012). The former study showed that microparticles enhance expression of OPN in spheroids and, surprisingly, the level of OPN is higher in soft spheroids than in stiff spheroids. However, to the authors' knowledge, the effect of spheroids stiffness on mineralisation has not been studied so far. Therefore, further research is needed to elucidate the relationship between matrix stiffness, cell shape, RhoA/ROCK pathway activity and osteogenic differentiation in spheroids.

It could be questioned whether the intensive mineral staining observed at day 14 in 2D conditions is a hallmark of the terminal stage of osteogenic differentiation, considering that the essential osteogenesis-inducing transcription factors were expressed at low levels throughout the observation time. A study performed by Boyan and co-workers (2002) showed that dexamethasone-induced foetal rat calvarial (FRC) cells yield mineral nodules staining positively in von Kossa histological staining. Furthermore, the in vitro produced bone-like matrix was analysed by Fourier-transformed infrared (FTIR) spectroscopy and the obtained spectrum was compared with the one of natural bone. Despite the presence of calcium compounds in the cell matrix, as evidenced by von Kossa staining, the cellderived minerals do not have the hydroxyapatite characteristic $\mathrm{Ca} / \mathrm{P}$ ratio and the mineral phase is not bound to the protein phase. The authors concluded that cell-derived minerals are dystrophic rather than physiologic because the mineralisation process occurred prematurely (Boyan et al., 2002). Bouet et al. (2015) examined the role of BSP on primary mouse calvaria cells (MCC). Knock-down of IBSP inhibits but does not block mineralisation of MCC in vitro, as analysed by von Kossa staining. These results confirmed the role of BSP as a factor necessary for efficient late-stage osteoblast differentiation in osteogenically committed cells. Therefore, positive histological staining for the mineral phase should not be treated as a sole marker of late differentiation stages and the extensive mineralisation observed in this study at day 14 in attachment-dependent cultures did not infer successful generation of mature osteoblasts. Expression of molecular markers, such as Runx2 and osterix transcription factors, as well as production of bone-mineral-binding non-collagenous matrix proteins should be also taken into account for the evaluation of osteogenic differentiation of ADSCs.

Adipogenic differentiation of ADSCs in 2D TCPS culture and 3D spheroids was compared by analysing the expression level of PPARG, CEBPA, FABP4 and $L P L$. The mean transcript level for all analysed genes was lower at both time points in spheroid culture. However, the differences in transcript level between 2D TCPS and 3D spheroids failed to reach statistical significance in any of the tested experimental conditions. Interestingly, literature data point out that BMSC adipogenesis, similar to osteogenesis, is stimulated by the spheroid environment (Wang et al., 2009). However, from the molecular point of view, adipogenesis and osteogenesis are considered to be opposing processes. The signalling pathways and molecular regulators that promote osteogenesis usually inhibit adipogenesis (Abdallah et al., 2015). However, in the present study, a significant effect of the 3D spheroid culture environment on adipogenic differentiation was not observed. Therefore, it is not possible to conclude that the altered osteogenic differentiation process observed in 3D spheroids was, at least partially, caused by the changes in adipogenic differentiation potential.

As mineral production was found to be inhibited in spheroids, late osteogenic differentiation events in $2 \mathrm{D}$ culture after pre-differentiation in spheroids were studied. The expression of osteocalcin peaked at day 14 of differentiation, with the expression level in spheroid pre-differentiated ADSCs higher than that in 2D pre-differentiated cells, despite being not statistically significant. Expression of osteocalcin was followed by mineralisation, where an equal mineral content was found for both predifferentiation conditions. This result indicated that although mineral production was inhibited in spheroids, the spheroid pre-differentiated cells retained the capability for mineral production in 2D culture. In fact, mineral deposition in ADSC cultures might be more efficient in attachmentdependent conditions, regardless of whether in 2D or 3D culture, as the polystyrene scaffolds were found to support mineralisation with an efficiency 
similar to that observed for 2D TCPS. Interestingly, in contrast to that in spheroid culture, the expression level of osterix was low at the 2D maturation stage, regardless of the applied pre-differentiation protocol. Considering the efficient osteocalcin and bone mineral production at this stage, a decrease in osterix expression might represent a switch between the pre- and mature osteoblast stage. Interestingly, Tsao et al. (2017) reported a direct relationship between osteocalcin level and bone mineral quality. Human BMSCs with osteocalcin gene knock-down show delayed deposition of beta-tricalcium phosphate and hydroxyapatite as analysed by Raman spectroscopy, indicating delayed bone mineral maturation. The time-dependent relation of osteocalcin expression and bone mineral production observed in the present study, supported this mechanism - bone mineral accumulation at day 21 was preceded by a peak in osteocalcin production. It is noteworthy that the delayed osteoblast maturation observed in continuous 3D spheroid culture was not prevalent - when ADSCs spheroids were disassociated, osteoblast maturation was equally efficient as in 2D TCPS. Furthermore, it would be interesting to compare the structure of cell-derived bone minerals from 2D TCPS at day 14 and 3D spheroid pre-differentiated culture at day 21. Future studies, utilising spectroscopic techniques, could address this question to elucidate if the 3D spheroid pre-differentiation procedure allows to obtain high-quality bone minerals.

The mechanism of the improved osteogenic commitment of ADSC in spheroids remains to be determined. However, it is possible to speculate about possible biological processes affected by the spheroid environment that may play some role. Collagenmediated integrin $\alpha 2 \beta 1$ signalling is indispensable for improved mineralisation in BMSC spheroids (Murphy et al., 2016). Therefore, the spheroid environment might improve the efficiency of binding collagen or other extracellular matrix proteins to integrins, which in turn could provide improved intracellular signalling through ERKs. Furthermore, gap-junction-mediated cell-to-cell communication is important for spheroid-induced osteogenesis, as ALP activity in osteogenically induced rat BMSCs is significantly down-regulated by $18 \alpha$-glycyrrhetinic acid (AGA), a gap junction inhibitor (Tang et al., 2010). Apart from gap junctions, adherens junctions may also play a role in spheroid-induced osteogenesis. Previous reports found that both $\mathrm{N}$-cadherin and OB-cadherin expression supports osteoblast marker gene expression and mineralisation in mouse osteoblast-enriched calvaria cultures (Di Benedetto et al., 2010). Furthermore, the levels of N-cadherin and OB-cadherin in osteogenically differentiated rat ADSCs are elevated in chitosan-derived spheroids as compared to monolayer culture (Hsu and Huang, 2013). Finally, the increased secretion of trophic factors in spheroids might contribute to improved osteogenesis, as BMP-2 expression is up-regulated 30-fold in human BMSC spheroids as compared to monolayer BMSCs (Kabiri et al., 2012). Nonetheless, further functional studies are needed to test if the presented hypotheses are valid for human ADSC osteogenesis in spheroids.

\section{Conclusions}

The present work provided evidence for the modulation of ADSC osteogenic differentiation by a 3D culture environment. Scaffold-free 3D spheroid culture increased the commitment of ADSCs into pre-osteoblasts as compared to that of 3D scaffold and monolayer conditions, but efficient osteoblast maturation and production of the mineralised extracellular matrix required an attachment substrate for the cells. The data presented might be used to improve the protocols for preparing ADSCs that are applied in the treatment of skeletal defects.

\section{Acknowledgments}

The work was supported by the National Centre for Research and Development, grant number STRATEGMED2/267976/13/NCBR/2015, and the Medical University of Warsaw, grant for young scientists number 1M15/PM11D/15.

The study was carried out with the use of Centre for Preclinical Research and Technology (CePT), Warsaw, Poland infrastructure, financed by the European Union - the European Regional Development Fund within the operational programme "Innovative economy" for 2007-2013.

\section{References}

Abbasi F, Ghanian MH, Baharvand $\mathrm{H}$, Vahidi B, Eslaminejad MB (2018) Engineering mesenchymal stem cell spheroids by incorporation of mechanoregulator microparticles. J Mech Behav Biomed Mater 84: 74-87.

Abdallah BM, Jafari A, Zaher W, Qiu W, Kassem M (2015) Skeletal (stromal) stem cells: an update on intracellular signaling pathways controlling osteoblast differentiation. Bone 70: 28-36.

Baraniak PR, Cooke MT, Saeed R, Kinney MA, Fridley KM, McDevitt TC (2012) Stiffening of human mesenchymal stem cell spheroid microenvironments induced by incorporation of gelatin microparticles. J Mech Behav Biomed Mater 11: 63-71.

Baraniak PR, McDevitt TC (2012) Scaffold-free culture of mesenchymal stem cell spheroids in suspension preserves multilineage potential. Cell Tissue Res 347: 701-711.

Bouet G, Bouleftour W, Juignet L, Linossier MT, Thomas M, Vanden-Bossche A, Aubin JE, Vico L, Marchat D, Malaval L (2015) The impairment of 
osteogenesis in bone sialoprotein (BSP) knockout calvaria cell cultures is cell density dependent. PLoS One 10: e0117402. DOI: 10.1371/journal.pone.0117402.

Boyan BD, Bonewald LF, Paschalis EP, Lohmann CH, Rosser J, Cochran DL, Dean DD, Schwartz Z, Boskey AL (2002) Osteoblast-mediated mineral deposition in culture is dependent on surface microtopography. Calcif Tissue Int 71: 519-529.

Bruderer M, Richards RG, Alini M, Stoddart MJ (2014) Role and regulation of RUNX2 in osteogenesis. Eur Cell Mater 28: 269-286.

Burns JS, Rasmussen PL, Larsen KH, Schroder HD, Kassem M (2010) Parameters in three-dimensional osteospheroids of telomerized human mesenchymal (stromal) stem cells grown on osteoconductive scaffolds that predict in vivo bone-forming potential. Tissue Eng Part A 16: 2331-2342.

Capulli M, Paone R, Rucci N (2014) Osteoblast and osteocyte: games without frontiers. Arch Biochem Biophys 561: 3-12.

Cheng NC, Chen SY, Li JR, Young TH (2013) Shortterm spheroid formation enhances the regenerative capacity of adipose-derived stem cells by promoting stemness, angiogenesis, and chemotaxis. Stem Cells Transl Med 2: 584-594.

Cheng NC, Wang S, Young TH (2012) The influence of spheroid formation of human adiposederived stem cells on chitosan films on stemness and differentiation capabilities. Biomaterials 33: 17481758.

Cho HH, Shin KK, Kim YJ, Song JS, Kim JM, Bae YC, Kim CD, Jung JS (2010) NF-kappaB activation stimulates osteogenic differentiation of mesenchymal stem cells derived from human adipose tissue by increasing TAZ expression. J Cell Physiol 223: 168177.

Coskun S, Chao H, Vasavada H, Heydari K, Gonzales N, Zhou X, de Crombrugghe B, Hirschi KK (2014) Development of the fetal bone marrow niche and regulation of HSC quiescence and homing ability by emerging osteolineage cells. Cell Rep 9: 581-590.

Di Benedetto A, Watkins M, Grimston S, Salazar V, Donsante C, Mbalaviele G, Radice GL, Civitelli R (2010) N-cadherin and cadherin 11 modulate postnatal bone growth and osteoblast differentiation by distinct mechanisms. J Cell Sci 123: 2640-2648.

Engler AJ, Sen S, Sweeney HL, Discher DE (2006) Matrix elasticity directs stem cell lineage specification. Cell 126: 677-689.

Erices A, Conget P, Minguell JJ (2000) Mesenchymal progenitor cells in human umbilical cord blood. Br J Haematol 109: 235-242.

Fennema E, Rivron N, Rouwkema J, van Blitterswijk C, de Boer J (2013) Spheroid culture as a tool for creating 3D complex tissues. Trends Biotechnol 31: 108-115.

Gabbay JS, Heller JB, Mitchell SA, Zuk PA, Spoon DB, Wasson KL, Jarrahy R, Benhaim P, Bradley JP (2006) Osteogenic potentiation of human adiposederived stem cells in a 3-dimensional matrix. Ann Plast Surg 57: 89-93.
Goodwin HS, Bicknese AR, Chien SN, Bogucki BD, Quinn CO, Wall DA (2001) Multilineage differentiation activity by cells isolated from umbilical cord blood: expression of bone, fat, and neural markers. Biol Blood Marrow Transplant 7: 581-588.

Guidotti S, Facchini A, Platano D, Olivotto E, Minguzzi M, Trisolino G, Filardo G, Cetrullo S, Tantini B, Martucci E, Facchini A, Flamigni F, Borzi RM (2013) Enhanced osteoblastogenesis of adiposederived stem cells on spermine delivery via betacatenin activation. Stem Cells Dev 22: 1588-1601.

Hao Z, Song Z, Huang J, Huang K, Panetta A, Gu Z, Wu J (2017) The scaffold microenvironment for stem cell based bone tissue engineering. Biomater Sci 5: 1382-1392.

Hildebrandt C, Buth H, Thielecke H (2011) A scaffold-free in vitro model for osteogenesis of human mesenchymal stem cells. Tissue Cell 43: 91-100.

Hishikawa K, Miura S, Marumo T, Yoshioka H, Mori Y, Takato T, Fujita T (2004) Gene expression profile of human mesenchymal stem cells during osteogenesis in three-dimensional thermoreversible gelation polymer. Biochem Biophys Res Commun 317: 1103-1107.

Ho SS, Keown AT, Addison B, Leach JK (2017) Cell migration and bone formation from mesenchymal stem cell spheroids in alginate hydrogels are regulated by adhesive ligand density. Biomacromolecules 18: 4331-4340.

Ho SS, Murphy KC, Binder BY, Vissers CB, Leach JK (2016) Increased survival and function of mesenchymal stem cell spheroids entrapped in instructive alginate hydrogels. Stem Cells Transl Med 5: 773-781.

Hsu SH, Huang GS (2013) Substrate-dependent Wnt signaling in MSC differentiation within biomaterial-derived 3D spheroids. Biomaterials 34: 4725-4738.

Jhala D, Vasita R (2015) A review on extracellular matrix mimicking strategies for an artificial stem cell niche. Polymer Reviews 55: 561-595.

Kabiri M, Kul B, Lott WB, Futrega K, Ghanavi P, Upton Z, Doran MR (2012) 3D mesenchymal stem/ stromal cell osteogenesis and autocrine signalling. Biochem Biophys Res Commun 419: 142-147.

Kalaszczynska I, Ruminski S, Platek AE, Bissenik I, Zakrzewski P, Noszczyk M, Lewandowska-Szumiel M (2013) Substantial differences between human and ovine mesenchymal stem cells in response to osteogenic media: how to explain and how to manage? Biores Open Access 2: 356-363.

Kale S, Biermann S, Edwards C, Tarnowski C, Morris M, Long MW (2000) Three-dimensional cellular development is essential for ex vivo formation of human bone. Nat Biotechnol 18: 954-958.

Khatiwala CB, Peyton SR, Putnam AJ (2006) Intrinsic mechanical properties of the extracellular matrix affect the behavior of pre-osteoblastic MC3T3-E1 cells. Am J Physiol Cell Physiol 290: C16401650. 
Knight E, Przyborski S (2015) Advances in 3D cell culture technologies enabling tissue-like structures to be created in vitro. J Anat 227: 746-756.

Lee JS, Lee JM, Im GI (2011) Electroporationmediated transfer of Runx2 and Osterix genes to enhance osteogenesis of adipose stem cells. Biomaterials 32: 760-768.

Li J, Liu X, Crook JM, Wallace GG (2017) Development of a porous 3D graphene-PDMS scaffold for improved osseointegration. Colloids Surf B Biointerfaces 159: 386-393.

Liu Q, Cen L, Zhou H, Yin S, Liu G, Liu W, Cao Y, Cui L (2009) The role of the extracellular signalrelated kinase signaling pathway in osteogenic differentiation of human adipose-derived stem cells and in adipogenic transition initiated by dexamethasone. Tissue Eng Part A 15: 3487-3497.

Liu TM, Lee EH (2013) Transcriptional regulatory cascades in Runx2-dependent bone development. Tissue Eng Part B Rev 19: 254-263.

Lough DM, Chambers C, Germann G, Bueno R, Reichensperger J, Swanson E, Dyer M, Cox L, Harrison C, Neumeister MW (2016) Regulation of ADSC osteoinductive potential using notch pathway inhibition and gene rescue: a potential on/ off switch for clinical applications in bone formation and reconstructive efforts. Plast Reconstr Surg 138: 642e-652e.

MacDonald C, Finlay DB, Jabed A, Glass M, Graham ES (2014) Development of positive control tissue for in situ hybridisation using Alvetex scaffolds. J Neurosci Methods 238: 70-77.

Malaval L, Wade-Gueye NM, Boudiffa M, Fei J, Zirngibl R, Chen F, Laroche N, Roux JP, Burt-Pichat B, Duboeuf F, Boivin G, Jurdic P, Lafage-Proust MH, Amedee J, Vico L, Rossant J, Aubin JE (2008) Bone sialoprotein plays a functional role in bone formation and osteoclastogenesis. J Exp Med 205: 1145-1153.

Mebarki M, Coquelin L, Layrolle P, Battaglia S, Tossou M, Hernigou P, Rouard H, Chevallier N (2017) Enhanced human bone marrow mesenchymal stromal cell adhesion on scaffolds promotes cell survival and bone formation. Acta Biomater 59: 94107.

Murphy KC, Fang SY, Leach JK (2014) Human mesenchymal stem cell spheroids in fibrin hydrogels exhibit improved cell survival and potential for bone healing. Cell Tissue Res 357: 91-99.

Murphy KC, Hoch AI, Harvestine JN, Zhou D, Leach JK (2016) Mesenchymal stem cell spheroids retain osteogenic phenotype through alpha2beta1 signaling. Stem Cells Transl Med 5: 1229-1237.

Nishio Y, Dong Y, Paris M, O'Keefe RJ, Schwarz EM, Drissi H (2006) Runx2-mediated regulation of the zinc finger Osterix/Sp7 gene. Gene 372: 62-70.

Paduano F, Marrelli M, Amantea M, Rengo C, Rengo S, Goldberg M, Spagnuolo G, Tatullo M (2017) adipose tissue as a strategic source of mesenchymal stem cells in bone regeneration: a topical review on the most promising craniomaxillofacial applications. Int J Mol Sci 18. pii: E2140. DOI: 10.3390/ijms18102140.
Pittenger MF, Mackay AM, Beck SC, Jaiswal RK, Douglas R, Mosca JD, Moorman MA, Simonetti DW, Craig S, Marshak DR (1999) Multilineage potential of adult human mesenchymal stem cells. Science 284: 143-147.

Romagnoli C, Zonefrati R, Galli G, Puppi D, Pirosa A, Chiellini F, Martelli FS, Tanini A, Brandi ML (2015) In vitro behavior of human adipose tissue-derived stem cells on poly(epsilon-caprolactone) film for bone tissue engineering applications. Biomed Res Int 2015: 323571. DOI: 10.1155/2015/323571.

Romanov YA, Svintsitskaya VA, Smirnov VN (2003) Searching for alternative sources of postnatal human mesenchymal stem cells: candidate MSC-like cells from umbilical cord. Stem Cells 21: 105-110.

Rumiński S, Ostrowska B, Jaroszewicz J, Skirecki T, Włodarski K, Święszkowski W, LewandowskaSzumieł M (2018) Three-dimensional printed polycaprolactone-based scaffolds provide an advantageous environment for osteogenic differentiation of human adipose-derived stem cells. J Tissue Eng Regen Med 12: e473-e485.

Schneider CA, Rasband WS, Eliceiri KW (2012) NIH Image to ImageJ: 25 years of image analysis. Nat Methods 9: 671-675.

Shafiee A, Seyedjafari E, Soleimani M, Ahmadbeigi N, Dinarvand P, Ghaemi N (2011) A comparison between osteogenic differentiation of human unrestricted somatic stem cells and mesenchymal stem cells from bone marrow and adipose tissue. Biotechnology Letters 33: 1257-1264.

Shakya AK, Kandalam U (2017) Three-dimensional macroporous materials for tissue engineering of craniofacial bone. Br J Oral Maxillofac Surg 55: 875891.

Steward AJ, Kelly DJ (2015) Mechanical regulation of mesenchymal stem cell differentiation. J Anat 227: 717-731.

Strzelecka-Kiliszek A, Mebarek S, Roszkowska M, Buchet R, Magne D, Pikula S (2017) Functions of Rho family of small GTPases and Rho-associated coiledcoil kinases in bone cells during differentiation and mineralization. Biochim Biophys Acta 1861: 1009-1023.

Tabatabaei Qomi R, Sheykhhasan M (2017) Adipose-derived stromal cell in regenerative medicine: a review. World J Stem Cells 9: 107-117.

Tang J, Peng R, Ding J (2010) The regulation of stem cell differentiation by cell-cell contact on micropatterned material surfaces. Biomaterials 31: 2470-2476.

Tsao YT, Huang YJ, Wu HH, Liu YA, Liu YS, Lee OK (2017) Osteocalcin mediates biomineralization during osteogenic maturation in human mesenchymal stromal cells. Int J Mol Sci 18. pii: E159. DOI: 10.3390/ ijms18010159.

Vishnubalaji R, Al-Nbaheen M, Kadalmani B, Aldahmash A, Ramesh T (2012) Comparative investigation of the differentiation capability of bonemarrow- and adipose-derived mesenchymal stem cells by qualitative and quantitative analysis. Cell Tissue Res 347: 419-427. 
Wang HS, Hung SC, Peng ST, Huang CC, Wei HM, Guo YJ, Fu YS, Lai MC, Chen CC (2004) Mesenchymal stem cells in the Wharton's jelly of the human umbilical cord. Stem Cells 22: 1330-1337.

Wang W, Itaka K, Ohba S, Nishiyama N, Chung UI, Yamasaki Y, Kataoka K (2009) 3D spheroid culture system on micropatterned substrates for improved differentiation efficiency of multipotent mesenchymal stem cells. Biomaterials 30: 2705-2715.

Xue R, Qian Y, Li L, Yao G, Yang L, Sun Y (2017) Polycaprolactone nanofiber scaffold enhances the osteogenic differentiation potency of various human tissue-derived mesenchymal stem cells. Stem Cell Res Ther 8: 148. DOI: 10.1186/s13287-017-0588-0.

Yamaguchi Y, Ohno J, Sato A, Kido H, Fukushima T (2014) Mesenchymal stem cell spheroids exhibit enhanced in-vitro and in-vivo osteoregenerative potential. BMC Biotechnol 14: 105. DOI: 10.1186/ s12896-014-0105-9.

Zhang T, Lin S, Shao X, Shi S, Zhang Q, Xue C, Lin Y, Zhu B, Cai X (2018) Regulating osteogenesis and adipogenesis in adipose-derived stem cells by controlling underlying substrate stiffness. J Cell Physiol 233: 3418-3428.

Zuk PA, Zhu M, Mizuno H, Huang J, Futrell JW, Katz AJ, Benhaim P, Lorenz HP, Hedrick MH (2001) Multilineage cells from human adipose tissue: implications for cell-based therapies. Tissue Eng 7: 211-228.

\section{Discussion with Reviewer}

Elena Della Bella: Can the authors comment in more detail why ADSCs grown as spheroids have the highest expression of osteogenic markers but show the lowest mineralisation? Which approach and which additional experiments would you suggest for explaining this result?

Authors: It would be interesting to study the minerals produced by ADSCs in 2D culture with spectroscopic techniques. This would allow to determine if the positive mineral staining observed already at day 14 was composed of natural-bonecharacteristic hydroxyapatite. Comparison of the minerals produced by cells pre-differentiated with the ones produced by spheroids would provide insight on wheatear the high expression of Runx2, osterix and BSP observed in spheroids improved the quality of the minerals deposited at a later stage of differentiation. Furthermore, the spheroid preparation method could be modified to include an attachment substrate, which, we believe, is needed for efficient mineralisation. For example, a simple RGD amino acid sequence or some type of nanoparticles (e.g. bioglass or graphene) could be used to stimulate the focal adhesion kinase (FAK)/ERK pathway in ADSCs and/or to provide a nucleation surface for the cell-produced bone mineral crystals. Finally, it is possible that the persistent expression of Runx2 inhibits late-stage differentiation in spheroids. Therefore, ADSCs could be transfected with an inducible shRNA vector targeting Runx2 transcript. After pre-differentiation of ADSCs, knock-down of Runx2 could be induced to check wheatear latestage differentiation and mineralisation would be enhanced.

Editor's note: The Scientific Editor responsible for this paper was Mauro Alini. 